\title{
Contribution of the Darwin operator to non-leptonic decays of heavy quarks
}

\author{
Alexander Lenz, Maria Laura Piscopo and Aleksey V. Rusov \\ Institute for Particle Physics Phenomenology, Durham University, \\ DH1 3LE Durham, U.K. \\ E-mail: alexander.lenz@uni-siegen.de, maria.piscopo@uni-siegen.de, \\ rusov@physik.uni-siegen.de
}

Abstract: We compute the Darwin operator contribution $\left(1 / m_{b}^{3}\right.$ correction) to the width of the inclusive non-leptonic decay of a $B$ meson $\left(B^{+}, B_{d}\right.$ or $\left.B_{s}\right)$, stemming from the quark flavour-changing transition $b \rightarrow q_{1} \bar{q}_{2} q_{3}$, where $q_{1}, q_{2}=u, c$ and $q_{3}=d, s$. The key ideas of the computation are the local expansion of the quark propagator in the external gluon field including terms with a covariant derivative of the gluon field strength tensor and the standard technique of the Heavy Quark Expansion (HQE). We confirm the previously known expressions of the $1 / m_{b}^{3}$ contributions to the semi-leptonic decay $b \rightarrow q_{1} \ell \bar{\nu}_{\ell}$, with $\ell=$ $e, \mu, \tau$ and of the $1 / m_{b}^{2}$ contributions to the non-leptonic modes. We find that this new term can give a sizeable correction of about $-4 \%$ to the non-leptonic decay width of a $B$ meson. For $B_{d}$ and $B_{s}$ mesons this turns out to be the dominant correction to the free b-quark decay, while for the $B^{+}$meson the Darwin term gives the second most important correction - roughly $1 / 2$ to $1 / 3$ of the phase space enhanced Pauli interference contribution. Due to the tiny experimental uncertainties in lifetime measurements the incorporation of the Darwin term contribution is crucial for precision tests of the Standard Model.

KEYwords: Heavy Quark Physics, Effective Field Theories, Nonperturbative Effects

ARXIV EPRINT: 2004.09527 


\section{Contents}

1 Introduction 1

2 Outline of the calculation 5

2.1 Contribution of two-quark operators up to order $1 / m_{b}^{3} \quad 5$

2.2 Role of the four-quark operators 9

3 Results 12

$\begin{array}{lll}4 & \text { Discussion and conclusion } & 15\end{array}$

$\begin{array}{lll}\text { A Expansion of the quark propagator in the external gluon field } & 17\end{array}$

$\begin{array}{ll}\text { B Complementary material to section } 2.2 & 19\end{array}$

$\begin{array}{ll}\text { C Contribution of four-quark operators at order } 1 / m_{b}^{3} & 21\end{array}$

D Coefficients of the dimension-three and of the chromo-magnetic operators

\section{Introduction}

The total decay rate of heavy hadrons can be described by the Heavy Quark Expansion (HQE), whose history goes back to the work of Shifman and Voloshin in the 1980ies [1, 2], ${ }^{1}$ as the decay of the free heavy quark plus corrections that are suppressed by inverse powers of the heavy quark mass. Since the $b$-quark mass is large compared to the typical hadronic scale, the corrections are expected to be small and hence, back in 1986, the following $b$-hadron lifetime ratios were expected [2]

$$
\left.\frac{\tau\left(B_{s}\right)}{\tau\left(B_{d}\right)}\right|^{\mathrm{HQE} 1986} \approx 1,\left.\quad \frac{\tau\left(B^{+}\right)}{\tau\left(B_{d}\right)}\right|^{\mathrm{HQE} 1986} \approx 1.1,\left.\quad \frac{\tau\left(\Lambda_{b}\right)}{\tau\left(B_{d}\right)}\right|^{\mathrm{HQE} 1986} \approx 1,
$$

which are in good agreement with the current experimental averages obtained by HFLAV (see the webupdate of ref. [4])

$$
\left.\frac{\tau\left(B_{s}\right)}{\tau\left(B_{d}\right)}\right|^{\mathrm{HFLAV} 2020}=0.998 \pm 0.004,\left.\quad \frac{\tau\left(B^{+}\right)}{\tau\left(B_{d}\right)}\right|^{\mathrm{HFLAV} 2020}=1.076 \pm 0.004,\left.\quad \frac{\tau\left(\Lambda_{b}\right)}{\tau\left(B_{d}\right)}\right|^{\mathrm{HFLAV} 2020}=0.969 \pm 0.006 .
$$

\footnotetext{
${ }^{1}$ For a more profound history see ref. [3].
} 


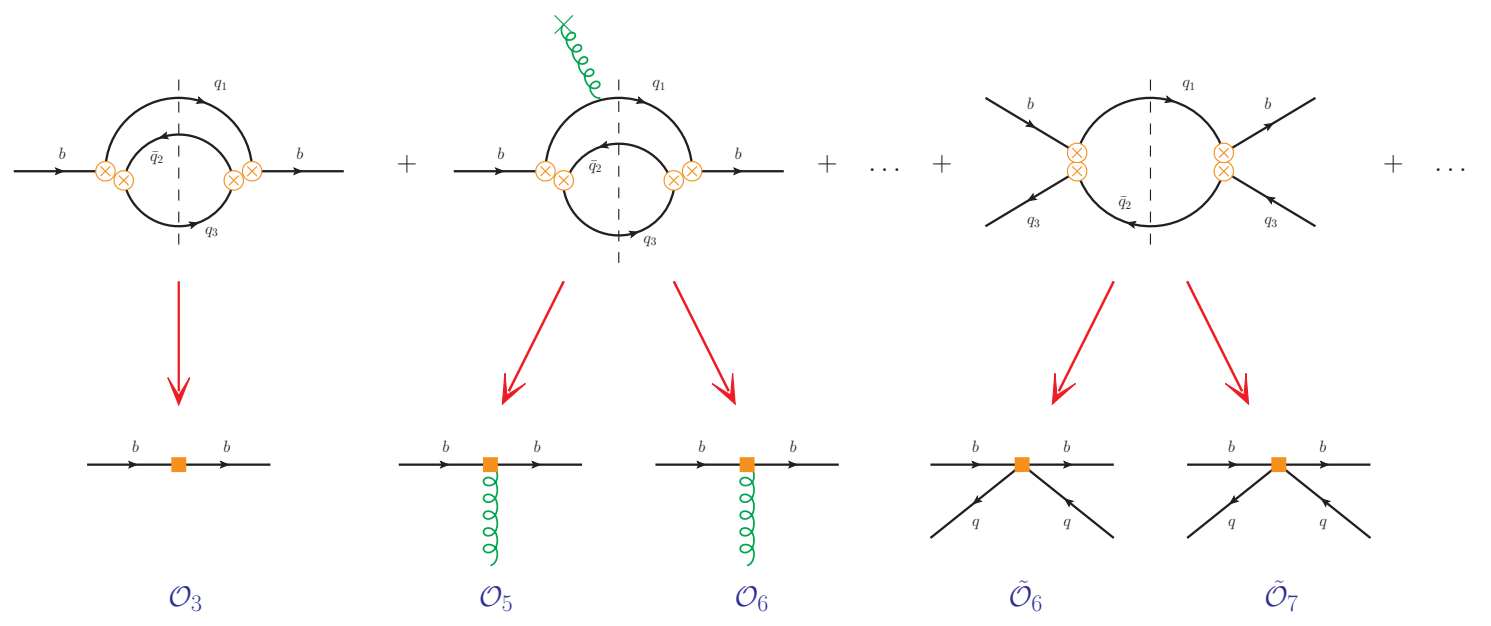

Figure 1. The diagrams describing contributions to the HQE in eq. (1.4). The crossed circles denote the $\Delta B=1$ operators $Q_{i}$ of the effective Hamiltonian while the squares denote the local $\Delta B=0$ operators $\mathcal{O}_{i}$ and $\tilde{\mathcal{O}}_{i}$. The two-loop and the phase space enhanced one-loop diagrams correspond respectively to the two-quark operators $\mathcal{O}_{i}$ and to the four-quark operators $\tilde{\mathcal{O}}_{i}$ in the HQE.

There has also been considerably progress on the theory side. The total width of a $B$ meson with mass $m_{B}$ and four-momentum $p_{B}^{\mu}$ is given by

$$
\Gamma(B)=\frac{1}{2 m_{B}} \sum_{X} \int_{\mathrm{PS}}(2 \pi)^{4} \delta^{(4)}\left(p_{B}-p_{X}\right)\left|\left\langle X\left(p_{X}\right)\left|\mathcal{H}_{\mathrm{eff}}\right| B\left(p_{B}\right)\right\rangle\right|^{2},
$$

where $\mathcal{H}_{\text {eff }}$ represents the effective weak Hamiltonian [5] describing all possible $b$-quark decays. $P S$ denotes the phase space integration and we have summed over all possible final states $X$ into which the $B$ meson can decay. Eq. (1.3) can be related, via the optical theorem, to the double insertion of the effective Hamiltonian and within the HQE framework one obtains

$$
\Gamma(B)=\Gamma_{3}+\Gamma_{5} \frac{\left\langle\mathcal{O}_{5}\right\rangle}{m_{b}^{2}}+\Gamma_{6} \frac{\left\langle\mathcal{O}_{6}\right\rangle}{m_{b}^{3}}+\ldots+16 \pi^{2}\left[\tilde{\Gamma}_{6} \frac{\left\langle\tilde{\mathcal{O}}_{6}\right\rangle}{m_{b}^{3}}+\tilde{\Gamma}_{7} \frac{\left\langle\tilde{\mathcal{O}}_{7}\right\rangle}{m_{b}^{4}}+\ldots\right]
$$

with the matrix elements of the $\Delta B=0$ operators $\left\langle\mathcal{O}_{Y}\right\rangle=\left\langle B\left(p_{B}\right)\left|\mathcal{O}_{Y}\right| B\left(p_{B}\right)\right\rangle$. The structure of eq. (1.4) is diagrammatically represented in figure 1. The matrix elements of the operators $\mathcal{O}_{i}$ and $\tilde{\mathcal{O}}_{i}$, denoting respectively two- and four-quark operators, are suppressed by $i-3$ powers of the heavy quark mass $m_{b}$. The coefficients $\Gamma_{i}$ and $\tilde{\Gamma}_{i}$ encode the corresponding short distance contributions. The leading term $\Gamma_{3}$ describes the free $b$-quark decay and does not contain any non-perturbative corrections - up to $\mathcal{O}\left(1 / m_{b}\right)$ the matrix element of the operator $\mathcal{O}_{3}=\bar{b} b$ is simply one with the appropriate normalisation. $\mathcal{O}_{5}$ refers to the dimension-five kinetic and chromo-magnetic operators, proportional to two covariant derivatives of the $b$-quark field. $\mathcal{O}_{6}$ includes the dimension-six Darwin and spinorbit operators with three covariant derivatives. So far the dependence on the spectator quark is only due to different values of the matrix elements and the coefficients $\Gamma_{3,5,6}$ are independent of the quark content of the $B$ meson. The four-quark operators are phasespace enhanced (as indicated by the factor $16 \pi^{2}$ ) and first arise at order $1 / m_{b}^{3}$. The 
possible topologies, specifically weak annihilation (WA), Pauli interference (PI) and weak exchange (WE), see figure 4, imply that the short distance coefficients are now dependent on the spectator quark. The dimension-seven operators $\tilde{\mathcal{O}}_{7}$ contain one covariant derivative compared to $\tilde{\mathcal{O}}_{6}$. Due to the larger phase space, it is typically expected that $\tilde{\mathcal{O}}_{6}$ gives the dominant contribution to the lifetime ratios $[1-3,6,7]$.

Currently $\Gamma_{3}$ is known at NLO-QCD [8-15] for non-leptonic decays. NNLO-QCD corrections have been computed for semi-leptonic decays [16-25] and for non-leptonic decays the massless case was determined in full QCD (i.e. no effective Hamiltonian was used) in ref. [26]. $\Gamma_{5}$ was determined at LO-QCD for both semi-leptonic and non-leptonic decays $[27-30]$ and we confirm these results. For the semi-leptonic modes even NLO-QCD corrections are available [31-33]. $\Gamma_{6}$ was first computed at LO-QCD in ref. [34] and recently the NLO-QCD corrections were determined in ref. [35], both for the semi-leptonic case only. $\tilde{\Gamma}_{6}$ is known at NLO-QCD for $B$-meson lifetimes [36, 37] and $D$ meson lifetimes [38], while $\tilde{\Gamma}_{7}$ is only known at LO-QCD [39].

This work presents the first determination of $\Gamma_{6}$ for non-leptonic decays. An interesting subtlety of the computation is mixing between four- and two-quark operators. Namely, at dimension-six the renormalised one-loop matrix elements of the operators $\tilde{\mathcal{O}}_{6}$ contribute to the coefficient of the Darwin operator through the diagram in figure 5, ensuring the cancellation of the infrared (IR) divergences that otherwise would appear in $\Gamma_{6}$. This feature has been intensively discussed for semi-leptonic decays [40-43] — also under the name of "intrinsic charm", see e.g. refs. [44, 45].

Finally, for numerical analysis, the values of the non-perturbative matrix elements $\left\langle\mathcal{O}_{Y}\right\rangle$ are needed. $\left\langle\mathcal{O}_{5}\right\rangle$ and $\left\langle\mathcal{O}_{6}\right\rangle$ can be extracted from fits to the semi-leptonic spectrum for the case of $B_{d}$ and $B^{+}$mesons, see e.g. ref. [46]. In the literature one can also find lattice determinations [47-51] and sum rule estimates [52-54] for these parameters. For the $\mathrm{SU}(3)_{F}$ violating ratios $\left\langle B_{s}\left|\mathcal{O}_{5,6}\right| B_{s}\right\rangle /\left\langle B_{d}\left|\mathcal{O}_{5,6}\right| B_{d}\right\rangle$ one can use the theory estimates from ref. [55]. The matrix elements of the four-quark operators $\left\langle\tilde{\mathcal{O}}_{6}\right\rangle$ have been determined by Heavy Quark Effective Theory (HQET) sum rules [56]. Violations of SU(3) $F$ are expected to yield visible effects and a calculation of these corrections with HQET sum rules following ref. [57] — is currently been performed [58]. Corresponding lattice results for the matrix elements of the four-quark operators would be highly desirable.

Taking all the currently known contributions into account we arrive at significant improvements compared to the pioneering work in 1986, and the 2019 status of lifetime predictions reads $[3,56]$

$$
\left.\frac{\tau\left(B_{s}\right)}{\tau\left(B_{d}\right)}\right|^{\mathrm{HQE} 2019}=1.0006 \pm 0.0025,\left.\quad \frac{\tau\left(B^{+}\right)}{\tau\left(B_{d}\right)}\right|^{\mathrm{HQE} 2019}=1.082_{-0.026}^{+0.022},\left.\quad \frac{\tau\left(\Lambda_{b}\right)}{\tau\left(B_{d}\right)}\right|^{\mathrm{HQE} 2019}=0.935 \pm 0.054
$$

which constitutes an impressive confirmation of the validity of the HQE.

The main motivations for this work are:

- In the case of the $\tau\left(B_{s}\right) / \tau\left(B_{d}\right)$ lifetime ratio several very pronounced cancellations are arising $[3,56,59-61]$ among the contributions of $\tilde{\mathcal{O}}_{6}$ and $\mathcal{O}_{5}$, which could in 
principle make this ratio sensitive to the contribution of $\mathcal{O}_{6}$ - if $\mathrm{SU}(3)_{F}$ violating corrections are large for this term, as indicated by ref. [55].

- It was found (see e.g. refs. [34, $41,55,62-64]$ ) that the $1 / m_{b}^{3}$ correction in semileptonic inclusive decays $B \rightarrow X_{c} \ell \bar{\nu}_{\ell}$ are of a similar size as the $1 / m_{b}^{2}$ terms due to enhanced Wilson coefficients. This could lead to a visible effect in $\tau\left(B_{s}\right) / \tau\left(B_{d}\right)$, in particular if all other contributions are cancelling to a high degree.

- As indicated in eq. (1.2) the experimental precision achieved so far is very high, enabling thus precision tests and an even higher precision seems to be achievable, see e.g. the two most recent results from LHCb [65] and ATLAS [66], which interestingly differ significantly. The theory precision should of course cope with these experimental advancements.

- According to the above arguments the lifetime ratio $\tau\left(B_{s}\right) / \tau\left(B_{d}\right)$ might thus provide a unique opportunity to test directly higher orders in the HQE that are otherwise just noise compared to the leading, numerically dominant contributions. In that respect this might also increase our insights on the assumptions of quark hadron duality, severely questioned around 20 years ago - mostly due to a very low experimental value of the $\Lambda_{b}$ lifetime, which seemed to be in severe conflict with the HQE expectation from eq. (1.1): the $2002 \mathrm{HFAG}$ average gives e.g. $\tau\left(\Lambda_{b}\right) / \tau\left(B_{d}\right)=0.798(34)$, more than $5 \sigma$ away from the current experimental value given by eq. (1.2). This signal for a violation of quark hadron duality was a clear false alarm. Moreover, the first measurement of the decay rate difference of neutral $B_{s}$ mesons, $\Delta \Gamma_{s}$, has excluded large duality violating effects, see e.g. the discussion in refs. [67, 68]. Smaller effects of duality violation can still appear and their potential size can be constrained by comparing experiment and theory for as many HQE observable as possible with a high precision, see e.g. ref. [69].

- Assuming the validity of quark hadron duality the $\tau\left(B_{s}\right) / \tau\left(B_{d}\right)$ lifetime ratio can be used to constrain invisible or hardly detectable $B$-meson decays, like $B_{s} \rightarrow \tau \tau$, at the per mille level, see e.g. refs. [70, 71] — see also ref. [72] for an alternative way to constrain the potential size of the $b s \tau \tau$ couplings.

We will not present updated lifetime ratio predictions in this work, but we will postpone a new numerical study until the $\mathrm{SU}(3)_{F}$ violation ratio of the Bag parameters is available, see ref. [58].

The paper is structured as follows: in section 2 we outline the main ingredients of the calculation: in section 2.1 we explain how the double insertion of the effective Hamiltonian and the subsequent expansion in the external soft gluon field are performed. Section 2.2 is devoted to describe mixing between two- and four-quark operators at order $1 / m_{b}^{3}$, which guarantees the cancellation of the IR divergences. Our results are presented in section 3 and we conclude in section 4 . The expansion of the quark propagator in the external gluon field is discussed in more detail in appendix A, supplement material to section 2.2 and 
section 3 can be found in appendix B and appendix C, respectively, and for completeness we also present the non-leptonic results for $\Gamma_{5}$ in appendix D.

\section{Outline of the calculation}

\subsection{Contribution of two-quark operators up to order $1 / m_{b}^{3}$}

According to the optical theorem, the total width for the inclusive non-leptonic decay of a $B$ meson induced at the quark level by the flavour-changing transition $b \rightarrow q_{1} \bar{q}_{2} q_{3}$ (with $q_{1}, q_{2}=u, c$ and $q_{3}=d, s$ ), can be computed from the discontinuity of the forward scattering matrix element:

$$
\Gamma_{\mathrm{NL}}(B)=\frac{1}{2 m_{B}} \operatorname{Im}\left\langle B\left(p_{B}\right)\left|i \int d^{4} x T\left\{\mathcal{L}_{\text {eff }}(x), \mathcal{L}_{\text {eff }}(0)\right\}\right| B\left(p_{B}\right)\right\rangle .
$$

The effective Lagrangian $\mathcal{L}_{\text {eff }}(x)$ reads

$$
\mathcal{L}_{\text {eff }}(x)=-\frac{4 G_{F}}{\sqrt{2}} V_{q_{1} b}^{*} V_{q_{2} q_{3}}\left[C_{1} Q_{1}(x)+C_{2} Q_{2}(x)\right]+\text { h.c. },
$$

where $G_{F}$ is the Fermi constant, $V_{q q^{\prime}}$ are the elements of the Cabibbo-Kobayashi-Maskawa (CKM) matrix [73, 74], $C_{1,2}\left(\mu_{1}\right)$ denote the Wilson coefficients at the renormalisation scale $\mu_{1} \sim m_{b}$ and $Q_{1,2}$ are the effective $\Delta B=1$ four-quark operators ${ }^{2}$

$$
\begin{aligned}
& Q_{1}=\left(\bar{q}_{1}^{i} \Gamma_{\mu} b^{i}\right)\left(\bar{q}_{3}^{j} \Gamma^{\mu} q_{2}^{j}\right), \\
& Q_{2}=\left(\bar{q}_{1}^{i} \Gamma_{\mu} b^{j}\right)\left(\bar{q}_{3}^{j} \Gamma^{\mu} q_{2}^{i}\right),
\end{aligned}
$$

with $i, j$ standing for colour indices and $\Gamma_{\mu} \equiv \gamma_{\mu}\left(1-\gamma_{5}\right) / 2$. In our notation, $Q_{1}$ is the colour singlet operator and $Q_{2}$ the colour rearranged one, opposite to e.g. the notation in ref. [5]. Three combinations of operators enter the decay rate in eq. (2.1): $Q_{1} \otimes Q_{1}, Q_{2} \otimes Q_{2}$ and $Q_{1} \otimes Q_{2}$. We use the completeness property of the colour matrices $t_{i j}^{a}{ }^{3}$

$$
t_{i j}^{a} t_{l m}^{a}=\frac{1}{2}\left(\delta_{i m} \delta_{j l}-\frac{1}{N_{c}} \delta_{i j} \delta_{l m}\right)
$$

to rewrite

$$
Q_{1} \otimes Q_{2}=\frac{1}{N_{c}}\left(Q_{1} \otimes Q_{1}\right)+2\left(Q_{1} \otimes T\right),
$$

where the colour octet operator $T$ is given by

$$
T=\left(\bar{q}_{1}^{i} \Gamma_{\mu} t_{i j}^{a} b^{j}\right)\left(\bar{q}_{3}^{l} \Gamma^{\mu} t_{l m}^{a} q_{2}^{m}\right) .
$$

Considering only the contribution of two-quark operators, the decay width takes the form

$$
\Gamma_{\mathrm{NL}}^{(2 \mathrm{q})}(B)=\left[C_{1}^{2} \Gamma_{11}^{(2 \mathrm{q})}+2 C_{1} C_{2}\left(\frac{1}{N_{c}} \Gamma_{11}^{(2 \mathrm{q})}+2 \Gamma_{1 T}^{(2 \mathrm{q})}\right)+C_{2}^{2} \Gamma_{22}^{(2 \mathrm{q})}\right],
$$

\footnotetext{
${ }^{2}$ We consider only current-current operators with large Wilson coefficients.

${ }^{3}$ The colour matrices satisfy the following relations: $\operatorname{Tr}\left[t^{a}\right]=0$ and $\operatorname{Tr}\left[t^{a}, t^{b}\right]=(1 / 2) \delta^{a b}$.
} 
where

$$
\begin{array}{r}
\Gamma_{11(1 T)}^{(2 q)}=-\frac{4 G_{F}^{2}\left|V_{q_{1} b}\right|^{2}\left|V_{q_{2} q_{3}}\right|^{2}}{m_{B}} \operatorname{Im}\left\langle B\left(p_{B}\right)\right| i \int d^{4} x \bar{b}(0) \Gamma_{\mu}\left(t^{a}\right) i S^{\left(q_{1}\right)}(0, x) \Gamma_{\nu} b(x) \\
\times \operatorname{Tr}\left[\Gamma^{\mu}\left(t^{a}\right) i S^{\left(q_{3}\right)}(0, x) \Gamma^{\nu} i S^{\left(q_{2}\right)}(x, 0)\right]\left|B\left(p_{B}\right)\right\rangle+(x \leftrightarrow 0) .
\end{array}
$$

Note that in the case of $\Gamma_{1 T}^{(2 \mathrm{q})}$ the two colour matrices $t^{a}$ appear on the r.h.s. of eq. (2.9). The corresponding expression for $\Gamma_{22}^{(2 \mathrm{q})}$ is obtained from that of $\Gamma_{11}^{(2 \mathrm{q})}$ by replacing $q_{1} \leftrightarrow q_{3}$ as it follows by Fierz-transforming the operator $Q_{2}$ given in eq. (2.4). To compute dimension-six contributions we need to expand first each of the quark propagators up to one covariant derivative of the gluon field strength tensor, respectively defined as $i D_{\mu}=i \partial_{\mu}+A_{\mu}(x),{ }^{4}$ and $G_{\mu \nu}=-i\left[i D_{\mu}, i D_{\nu}\right]$. In appendix A we derive the Fourier transform of the quark propagator in the soft external gluon field

$$
S(x, 0)=\int \frac{d^{4} k}{(2 \pi)^{4}} e^{-i k x} S(k),
$$

where

$$
\begin{aligned}
S(k)= & \frac{\not k+m}{k^{2}-m^{2}}+\frac{1}{2\left(k^{2}-m^{2}\right)^{2}}\left(-m G_{\rho \mu} \sigma^{\rho \mu}+2 \tilde{G}_{\rho \mu} k^{\rho} \gamma^{\mu} \gamma^{5}\right) \\
& +\frac{2}{3} \frac{D_{\rho} G^{\rho \mu}}{\left(k^{2}-m^{2}\right)^{2}}\left(\gamma_{\mu}-\frac{(\not k+2 m) k_{\mu}}{k^{2}-m^{2}}\right)-\frac{2}{3} \frac{D_{\nu} G_{\rho \mu} k^{\nu} k^{\rho} \gamma^{\mu}}{\left(k^{2}-m^{2}\right)^{3}} \\
& +\frac{2}{3} \frac{m D_{\nu} G_{\rho \mu}}{\left(k^{2}-m^{2}\right)^{3}}\left(\gamma^{\rho} \gamma^{\mu} k^{\nu}-\gamma^{\mu} \gamma^{\nu} k^{\rho}\right)+2 i \frac{D_{\nu} \tilde{G}_{\rho \mu} k^{\nu} k^{\rho} \gamma^{\mu} \gamma^{5}}{\left(k^{2}-m^{2}\right)^{3}}+\ldots
\end{aligned}
$$

In the previous equation, $\tilde{G}_{\rho \mu}=(1 / 2) \epsilon_{\rho \mu \sigma \eta} G^{\sigma \eta}$ denotes the dual field strength tensor, with $\epsilon_{\rho \mu \sigma \eta}$ being the Levi-Civita tensor and $D_{\rho} G_{\mu \nu}=-\left[i D_{\rho},\left[i D_{\mu}, i D_{\nu}\right]\right]$. From eq. (2.11) it follows that the quark propagator can be split up according to its colour structure as

$$
S_{i j}(k)=S^{(0)}(k) \delta_{i j}+S^{(1) a}(k) t_{i j}^{a},
$$

where $S^{(0)}(k)$ denotes the free quark propagator and $S^{(1) a}(k)$ absorbs contributions with one gluon field (for more details see appendix A, also the original refs. [28, 29]). This representation allows a straightforward treatment of colour in eq. (2.9). In case of $Q_{1} \otimes Q_{1}$ contribution, the colour flow factorises between the $\left(b q_{1}\right)$-quark line and the $\left(q_{2} q_{3}\right)$-loop, therefore one can only expand the propagator of $q_{1}$ up to terms linear in $t^{a}$, see l.h.s. of figure 2. For the $Q_{2} \otimes Q_{2}$ combination the trace over colour indices involves the $q_{1}$ - and $q_{2}$-quark propagators and only the gluon radiation from $q_{3}$ is non-vanishing, see r.h.s. of figure 2. Finally, in the case of the $Q_{1} \otimes T$ contribution, the colour flow forces the gluon to be radiated off the $\left(q_{2} q_{3}\right)$-loop. Any interference term would be $\mathcal{O}\left(1 / m_{b}^{4}\right)$, and it is thus sufficient to expand independently each of the two quark propagators in the loop (figure 3 ).

Using translation invariance of the quark field to write $b(x)=e^{-i p x} b(0)$, with $p^{\mu}$ being the $b$-quark four-momentum, and after performing $x$ - and momentum integration,

\footnotetext{
${ }^{4}$ Note that the coupling constant $g_{s}$ is absorbed in the definition of $A_{\mu}=A_{\mu}^{a} t^{a}$.
} 

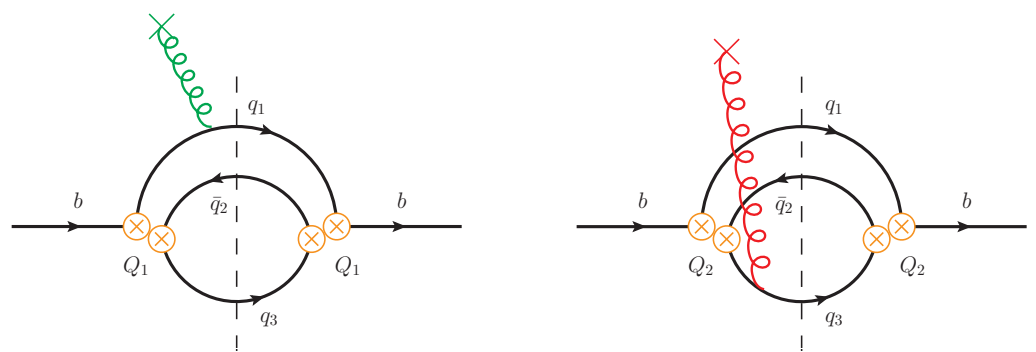

Figure 2. The two-loop diagrams describing power corrections up to dimension-six from $Q_{1} \otimes Q_{1}$ (left) and $Q_{2} \otimes Q_{2}$ (right) contributions.
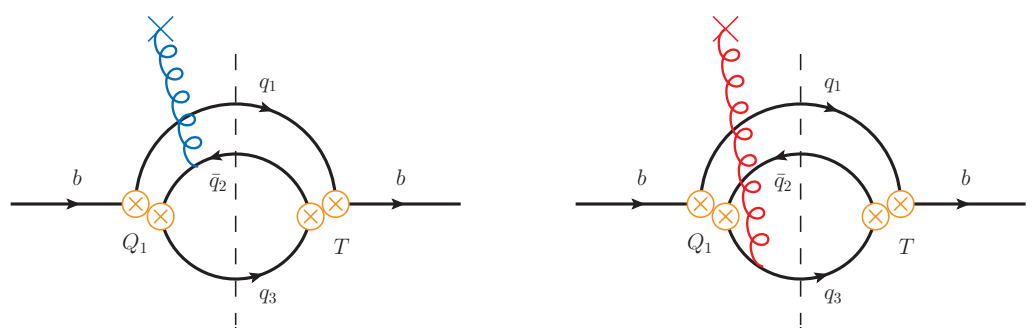

Figure 3. The two-loop diagrams describing power corrections up to dimension-six from $Q_{1} \otimes T$ contribution.

we obtain two-loop tensor integrals of possible rank $r=1, \ldots, 4$. These are decomposed in terms of rank $r$ tensors built with the metric tensor $g^{\mu \nu}$ and the external momentum $p^{\mu}$. The corresponding coefficients represent scalar integrals of the type

$$
\int \frac{d^{4} k_{1}}{(2 \pi)^{4}} \int \frac{d^{4} k_{2}}{(2 \pi)^{4}} \frac{f\left(p, k_{1}, k_{2}\right)}{\left[k_{1}^{2}-m_{1}^{2}\right]^{n_{1}}\left[k_{2}^{2}-m_{2}^{2}\right]^{n_{2}}\left[\left(p-k_{1}-k_{2}\right)^{2}-m_{3}^{2}\right]^{n_{3}}},
$$

where $f\left(p, k_{1}, k_{2}\right)$ is a function of all the possible scalar products. The integrals in eq. (2.13) are reduced by means of the integration-by-parts (IBP) technique to a linear combination of master integrals. The IBP reduction is implemented using the Mathematica package LiteRed $[75,76]$. The discontinuity of the master integrals can be straightforwardly obtained from the imaginary part of the scalar sunset diagram $S\left(s ; m_{1}, m_{2}, m_{3}\right)$, defined by eq. (2.13) with $f=1$ and $n_{1}=n_{2}=n_{3}=1$ and given by, ${ }^{5}$ see ref. [77]

$$
\operatorname{Im} S\left(s ; m_{1}, m_{2}, m_{3}\right)=\frac{1}{256 \pi^{3}} \int_{\left(m_{2}+m_{3}\right)^{2}}^{\left(\sqrt{s}-m_{1}\right)^{2}} d t \frac{\sqrt{\lambda\left(t, m_{2}^{2}, m_{3}^{2}\right) \lambda\left(s, t, m_{1}^{2}\right)}}{t s} .
$$

In the previous equation, $s=p^{2} \geq\left(m_{1}+m_{2}+m_{3}\right)^{2}$ and $\lambda(a, b, c) \equiv a^{2}+b^{2}+c^{2}-$ $2(a b+b c+a c)$ is the Källen function. One can easily compute analytically the integral in eq. (2.14) for two non-vanishing masses, while in the case of three non-vanishing masses the complexity highly increases and the solution involves elliptic functions, see e.g. refs. [7881]. We emphasize that we set $d=4$ from the beginning since the discontinuity of the

\footnotetext{
${ }^{5}$ For instance, the discontinuity of the master integral with $f=1, n_{1}=2$ and $n_{2}=n_{3}=1$ can be obtained by differentiating eq. (2.14) with respect to $m_{1}^{2}$, etc.
} 
diagrams at LO-QCD does not develop any ultraviolet divergences. On the other side, the gluon emission off a light quark propagator $(q=u, d$ or $s)$ gives rise, at dimension-six, to infrared logarithmic divergences of the type $\log \left(m_{q}^{2} / m_{b}^{2}\right)$ which are cancelled by the renormalised one-loop matrix element of the corresponding four-quark operators, as shown in detail in section 2.2. Here we limit ourselves to state that for the computation of most of the diagrams of interest it is always possible to set one mass to zero and obtain an analytic expression for all the master integrals. This is not the case only for the gluon emission from the $s$ quark in the $b \rightarrow c \bar{c} s$ transition, where we need to keep all masses finite in order to regularise the infrared divergence.

After computing the two-loop integrals we are left with the following matrix elements

$$
\begin{aligned}
& \left\langle B\left(p_{B}\right)\left|\bar{b}_{v}(0) \mathcal{F}(p) b_{v}(0)\right| B\left(p_{B}\right)\right\rangle, \\
& \left\langle B\left(p_{B}\right)\left|\bar{b}_{v}(0) \mathcal{F}_{\mu \nu}(p)\left(i D^{\mu}\right)\left(i D^{\nu}\right) b_{v}(0)\right| B\left(p_{B}\right)\right\rangle, \\
& \left\langle B\left(p_{B}\right)\left|\bar{b}_{v}(0) \mathcal{F}_{\mu \nu \rho}(p)\left(i D^{\mu}\right)\left(i D^{\nu}\right)\left(i D^{\rho}\right) b_{v}(0)\right| B\left(p_{B}\right)\right\rangle,
\end{aligned}
$$

where $\mathcal{F}(p), \mathcal{F}_{\mu \nu}(p)$ and $\mathcal{F}_{\mu \nu \rho}(p)$ are functions of the quark masses and of the external momentum $p^{\mu}$. We find that at order $1 / m_{b}^{2}$ only the expansion of the anti-quark propagator contributes (in agreement with refs. [27-30]) and therefore the matrix element of dimensionfive, eq. (2.16), vanishes in the case of both $Q_{1} \otimes Q_{1}$ and $Q_{2} \otimes Q_{2}$ contributions but not for $Q_{1} \otimes Q_{2}$. On the other hand, the matrix elements of dimension-three, eq. (2.15), and of dimension-six, eq. (2.17), are non-vanishing for all three combinations of $\Delta B=1$ operators. Following the standard technique of the HQE we decompose the $b$-quark momentum as

$$
p^{\mu}=m_{b} v^{\mu}+i D^{\mu}
$$

where $v^{\mu}$ represents the four-velocity of the $B$ meson and $D^{\mu}$ accounts for the soft interaction with the spectator quark. We have used the phase redefinition

$$
b(x)=e^{-i m_{b} v \cdot x} b_{v}(x),
$$

to remove the large fraction of the $b$-field momentum, at $x=0$ we trivially get $b(0)=b_{v}(0)$. We then expand eqs. (2.15)-(2.17) in the small quantity $D^{\mu} / m_{b}$. Note that in the matrix elements with three covariant derivatives we can safely set $p^{2}=m_{b}^{2}$ neglecting corrections $\mathcal{O}\left(1 / m_{b}^{4}\right)$. The order of the covariant derivatives is fixed by

$$
p^{\mu_{1}} p^{\mu_{2}} \ldots p^{\mu_{n}}=\frac{1}{n !} \sum_{\sigma \in \mathcal{S}_{n}} p^{\sigma\left(\mu_{1}\right)} p^{\sigma\left(\mu_{2}\right)} \ldots p^{\sigma\left(\mu_{n}\right)}
$$

where $\mathcal{S}_{n}$ is the symmetric group of permutation of $n$ elements. This leads to a systematic expansion of eq. (2.9), schematically

$$
a\left\langle\bar{b}_{v} b_{v}\right\rangle+b_{\mu}\left\langle\bar{b}_{v}\left(i D^{\mu}\right) b_{v}\right\rangle+c_{\mu \nu}\left\langle\bar{b}_{v}\left(i D^{\mu}\right)\left(i D^{\nu}\right) b_{v}\right\rangle+d_{\mu \nu \rho}\left\langle\bar{b}_{v}\left(i D^{\mu}\right)\left(i D^{\nu}\right)\left(i D^{\rho}\right) b_{v}\right\rangle+\ldots
$$

where $a, b_{\mu}, c_{\mu \nu}, d_{\mu \nu \rho}$ are now only functions of the quark masses and of the four-velocity $v^{\mu}$. 
Finally, the matrix elements in eq. (2.21) admit a series expansion in powers of $1 / m_{b}$ given explicitly in ref. [62], ${ }^{6}$ from which we can readily obtain the coefficients of $\mu_{\pi}^{2}, \mu_{G}^{2}$ and of $\rho_{D}^{3}, \rho_{L S}^{3}$, which are defined as

$$
\begin{aligned}
2 m_{B} \mu_{\pi}^{2}(B) & =-\left\langle B\left(p_{B}\right)\left|\bar{b}_{v}\left(i D_{\mu}\right)\left(i D^{\mu}\right) b_{v}\right| B\left(p_{B}\right)\right\rangle, \\
2 m_{B} \mu_{G}^{2}(B) & =\left\langle B\left(p_{B}\right)\left|\bar{b}_{v}\left(i D_{\mu}\right)\left(i D_{\nu}\right)\left(-i \sigma^{\mu \nu}\right) b_{v}\right| B\left(p_{B}\right)\right\rangle, \\
2 m_{B} \rho_{D}^{3}(B) & =\left\langle B\left(p_{B}\right)\left|\bar{b}_{v}\left(i D_{\mu}\right)(i v \cdot D)\left(i D^{\mu}\right) b_{v}\right| B\left(p_{B}\right)\right\rangle, \\
2 m_{B} \rho_{L S}^{3}(B) & =\left\langle B\left(p_{B}\right)\left|\bar{b}_{v}\left(i D_{\mu}\right)(i v \cdot D)\left(i D_{\nu}\right)\left(-i \sigma^{\mu \nu}\right) b_{v}\right| B\left(p_{B}\right)\right\rangle,
\end{aligned}
$$

with $\sigma_{\mu \nu}=(i / 2)\left[\gamma_{\mu}, \gamma_{\nu}\right]$. As already stressed, the numerical values of the non-perturbative parameters above depend on the spectator quark in the $B$ meson.

We then arrive at the following form of eq. (2.8)

$$
\begin{aligned}
\Gamma_{\mathrm{NL}}^{(2 \mathrm{q})}(B)= & \Gamma_{0}\left[\left(3 C_{1}^{2}+2 C_{1} C_{2}+3 C_{2}^{2}\right) \mathcal{C}_{0}^{\left(q_{1} \bar{q}_{2} q_{3}\right)}\left(1-\frac{\mu_{\pi}^{2}(B)}{2 m_{b}^{2}}\right)\right. \\
& +\left(3 C_{1}^{2} \mathcal{C}_{G, 11}^{\left(q_{1} \bar{q}_{2} q_{3}\right)}+2 C_{1} C_{2} \mathcal{C}_{G, 12}^{\left(q_{1} \bar{q}_{2} q_{3}\right)}+3 C_{2}^{2} \mathcal{C}_{G, 22}^{\left(q_{1} \bar{q}_{2} q_{3}\right)}\right) \frac{\mu_{G}^{2}(B)}{m_{b}^{2}} \\
& \left.+\left(3 C_{1}^{2} \mathcal{C}_{D, 11}^{\left(q_{1} \bar{q}_{2} q_{3}\right)}+2 C_{1} C_{2} \mathcal{C}_{D, 12}^{\left(q_{1} \bar{q}_{2} q_{3}\right)}+3 C_{2}^{2} \mathcal{C}_{D, 22}^{\left(q_{1} \bar{q}_{2} q_{3}\right)}\right) \frac{\rho_{D}^{3}(B)}{m_{b}^{3}}\right]
\end{aligned}
$$

where

$$
\Gamma_{0}=\frac{G_{F}^{2} m_{b}^{5}}{192 \pi^{3}}\left|V_{q_{1} b}\right|^{2}\left|V_{q_{2} q_{3}}\right|^{2}
$$

In eq. (2.24), $\mathcal{C}_{0}^{\left(q_{1} \bar{q}_{2} q_{3}\right)}$ refers to the partonic-level coefficient, while $\mathcal{C}_{G, n m}^{\left(q_{1} \bar{q}_{2} q_{3}\right)}$ and $\mathcal{C}_{D, n m}^{\left(q_{1} \bar{q}_{2} q_{3}\right)}$, $n m=\{11,12,22\}$, denote the coefficients of the chromo-magnetic and of the Darwin operators, respectively. The upper index $\left(q_{1} \bar{q}_{2} q_{3}\right)$ indicates the decay mode, e.g. $(c \bar{u} d)$ corresponds to the $b \rightarrow c \bar{u} d$ transition. Since we neglect the masses of the up, down and strange quark, all the coefficients in eq. (2.24) are functions of at most one dimensionless mass parameter $\rho=m_{c}^{2} / m_{b}^{2}$, apart from $\mathcal{C}_{D, m n}^{\left(q_{1} \bar{q}_{2} q_{3}\right)}$, where the dependence on the light quark mass $m_{q}=m_{u, d, s}$ is still present in the form of divergent logarithms $\log \left(m_{q}^{2} / m_{b}^{2}\right)$. The cancellation of these terms is discussed in the next subsection. The complete LO-QCD expressions for $\mathcal{C}_{0}^{\left(q_{1} \bar{q}_{2} q_{3}\right)}$ and $\mathcal{C}_{G, n m}^{\left(q_{1} \bar{q}_{2} q_{3}\right)}$ can be found in appendix D. We point out that the adopted definition for the non-perturbative parameters in eqs. (2.22), (2.23) implies that the coefficient of $\rho_{L S}^{3}$ is found to vanish for all the $\Delta B=1$ operator combinations.

\subsection{Role of the four-quark operators}

At order $1 / m_{b}^{3}$ the gluon radiation from a light quark leads to IR divergences, namely

$$
\mathcal{C}_{D, n m}^{\left(q_{1} \bar{q}_{2} q_{3}\right)}=\mathcal{R}_{n m}^{\left(q_{1} \bar{q}_{2} q_{3}\right)}+\mathcal{D}_{n m}^{\left(q_{1} \bar{q}_{2} q_{3}\right)}\left(\log \left(\frac{m_{q}^{2}}{m_{b}^{2}}\right)\right)
$$

\footnotetext{
${ }^{6}$ For reference we quote the expansion of the dimension-three matrix element up to order $1 / m_{b}^{3}$, $\left\langle B\left(p_{B}\right)\left|\bar{b}_{v} b_{v}\right| B\left(p_{B}\right)\right\rangle=2 m_{B}\left(1-\frac{\mu_{\pi}^{2}(B)-\mu_{G}^{2}(B)}{2 m_{b}^{2}}\right)$.
} 

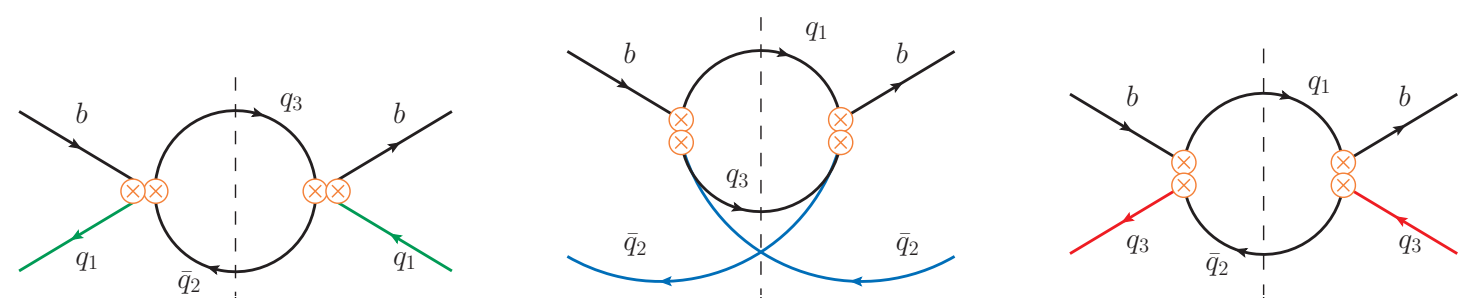

Figure 4. One-loop diagrams corresponding, from left to right, to the WA, PI and WE topology.

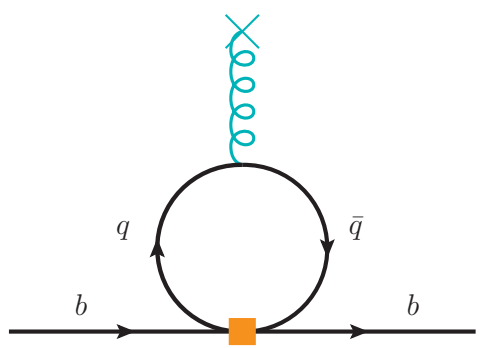

Figure 5. Diagram contributing to mixing between four- and two-quark operators at dimension-six.

where $\mathcal{R}_{n m}^{\left(q_{1} \bar{q}_{2} q_{3}\right)}$ are finite functions and $\mathcal{D}_{n m}^{\left(q_{1} \bar{q}_{2} q_{3}\right)}\left(\log \left(m_{q}^{2} / m_{b}^{2}\right)\right)$ absorb the contribution of the divergent logarithms, the latter are listed in appendix B. As discussed already in ref. [40], logarithmic infrared divergences signal mixing between operators of the same dimension. To see this in more detail, we start again from eq. $(2.1),{ }^{7}$

$$
\Gamma_{\mathrm{NL}}(B)=\frac{1}{2 m_{B}}\left\langle B\left(p_{B}\right)|\operatorname{Im} \hat{T}| B\left(p_{B}\right)\right\rangle,
$$

with the transition operator $\hat{T}$ being the time-ordered product of the double insertion of the effective Lagrangian. The expansion in inverse powers of $m_{b}$ allows to express $\hat{T}$ in terms of new local effective operators. At dimension-six one has ${ }^{8}$ :

$$
\operatorname{Im} \hat{T}^{(d=6)}=\Gamma_{0} C\left(\mu_{1}\right)\left[\mathcal{C}_{\rho_{D}}\left(\mu_{1}, \mu_{0}\right) \frac{\mathcal{O}_{\rho_{D}}\left(\mu_{0}\right)}{m_{b}^{3}}+\sum_{q=u, d, s} \sum_{i=1}^{4} \mathcal{C}_{6, i}^{(q)}\left(\mu_{1}, \mu_{0}\right) \frac{\tilde{\mathcal{O}}_{6, i}^{(q)}\left(\mu_{0}\right)}{m_{b}^{3}}\right]
$$

where $C\left(\mu_{1}\right)=\left\{3 C_{1}^{2}, 2 C_{1} C_{2}, 3 C_{2}^{2}\right\}$ and $\mu_{1}$ is the renormalisation scale at which the $\Delta B=$ 1 Wilson coefficients of the weak Hamiltonian are determined. This scale arises also in loop corrections to the diagrams given in the upper line of figure 1. Up to the calculated order in the strong coupling the $\mu_{1}$ dependence will cancel among these two sources. The second scale $\mu_{0}\left(\Lambda_{\mathrm{QCD}} \ll \mu_{0} \leq \mu_{1} \sim m_{b}\right)$ indicates the new factorisation scale of the $\Delta B=0$ operators emerging in the $\mathrm{HQE}$. The $\mu_{0}$ dependence will cancel among loop corrections to the diagrams given in the lower line of figure 1 and the scale dependence of the $\Delta B=0$ operators.

\footnotetext{
${ }^{7}$ For brevity we omit the indices $\left(q_{1} \bar{q}_{2} q_{3}\right)$ and $(n m)$, although they must be always understood.

${ }^{8}$ In principle the sum in eq. (2.28) includes also the $c$-quark but for $m_{b} \sim m_{c} \gg \Lambda_{Q C D}$ the four-quark operators $\tilde{\mathcal{O}}_{6, i}^{(c)}$ are not relevant for the further discussion, see for more details ref. [44].
} 
The l.h.s. of eq. (2.28) includes the contributions given by the two-loop diagrams in figure 2 and figure 3 , which give rise directly to the Darwin operator $\mathcal{O}_{\rho_{D}},{ }^{9}$

$$
\mathcal{O}_{\rho_{D}}=\bar{b}_{v}\left(i D_{\mu}\right)(i v \cdot D)\left(i D^{\mu}\right) b_{v}
$$

with the corresponding coefficient $\mathcal{C}_{D}\left(\mu_{1}\right)$ - see eq. (2.24). Since we are only working at LO-QCD, we do not find any explicit $\mu_{1}$-dependence at this stage. The l.h.s. of eq. (2.28) receives also contributions by the one-loop diagrams depicted in figure 4, corresponding to the weak-annihilation, Pauli interference and weak-exchange topologies. The coefficients $\mathcal{C}_{6, i}^{(q)}\left(\mu_{1}, \mu_{0}\right)$ do not develop any divergences at LO-QCD $[6,7]$ hence there is no explicit $\mu_{1}$ - and $\mu_{0}$-dependence; it is however present in the NLO-QCD corrections determined in refs. [36, 37, 82]. We refer to appendix B for explicit expressions at LO-QCD. Integrating out these one-loop diagrams leads to the following $\Delta B=0$ four-quark operators: ${ }^{10}$

$$
\begin{array}{ll}
\tilde{\mathcal{O}}_{6,1}^{(q)}=\left(\bar{b}_{v}^{i} \gamma_{\mu}\left(1-\gamma_{5}\right) q^{i}\right)\left(\bar{q}^{j} \gamma^{\mu}\left(1-\gamma_{5}\right) b_{v}^{j}\right), & \tilde{\mathcal{O}}_{6,2}^{(q)}=\left(\bar{b}_{v}^{i} \psi\left(1-\gamma_{5}\right) q^{i}\right)\left(\bar{q}^{j} \psi\left(1-\gamma_{5}\right) b_{v}^{j}\right), \\
\tilde{\mathcal{O}}_{6,3}^{(q)}=\left(\bar{b}_{v}^{i} \gamma_{\mu}\left(1-\gamma_{5}\right) q^{j}\right)\left(\bar{q}^{j} \gamma^{\mu}\left(1-\gamma_{5}\right) b_{v}^{i}\right), & \tilde{\mathcal{O}}_{6,4}^{(q)}=\left(\bar{b}_{v}^{i} \psi\left(1-\gamma_{5}\right) q^{j}\right)\left(\bar{q}^{j} \psi\left(1-\gamma_{5}\right) b_{v}^{i}\right) .
\end{array}
$$

The four-quark and the Darwin operators mix under operator renormalisation already at LO-QCD, through the diagram in figure 5. In dimensional regularisation with $d=4-2 \epsilon$ a factor of $\mu^{2 \epsilon}$ must be included in order to compensate the mismatch in the dimension of $\tilde{\mathcal{O}}_{6, i}^{(q)}$ and $\mathcal{O}_{\rho_{D}}$, leading to explicitly $\mu$-dependent renormalisation constants and thus a non-vanishing anomalous dimension matrix. The renormalised one-loop matrix element of $\tilde{\mathcal{O}}_{6, i}^{(q)}$ in the presence of a soft background gluon field, in NDR and in the $\overline{\mathrm{MS}}$ scheme reads:

$$
\left\langle\tilde{\mathcal{O}}_{6, i}^{(q)}\right\rangle^{\text {ren }}=\frac{a_{i}}{12 \pi^{2}}\left[\log \left(\frac{\mu^{2}}{m_{q}^{2}}\right)+b_{i}\right]\left\langle\mathcal{O}_{\rho_{D}}\right\rangle+\mathcal{O}\left(\frac{1}{m_{b}}\right)
$$

with $a_{1}=2, a_{2}=-1, a_{3}=a_{4}=0$ and $b_{1}=-1$ and $b_{2}=0$. Note that the presence of the constant term $b_{i}$ depends on the choice of the operator basis in eqs. (2.30), (2.31), see for instance refs. $[42,83]$. At the matching scale $\mu=m_{b}$, we obtain

$$
\mathcal{C}_{\rho_{D}}\left(\mu_{1}, m_{b}\right)\left\langle\mathcal{O}_{\rho_{D}}\left(m_{b}\right)\right\rangle=\mathcal{C}_{D}\left(\mu_{1}\right)\left\langle\mathcal{O}_{\rho_{D}}\left(m_{b}\right)\right\rangle-\sum_{q=u, d, s} \sum_{i=1}^{2} \mathcal{C}_{6, i}^{(q)}\left(\mu_{1}, m_{b}\right)\left\langle\tilde{\mathcal{O}}_{6, i}^{(q)}\left(m_{b}\right)\right\rangle^{\mathrm{ren}},
$$

where the coefficient $\mathcal{C}_{D}$ has the divergent logarithmic dependence shown in eq. (2.26). From it follows that on the r.h.s. of eq. (2.33) all the logarithms $\log \left(m_{q}^{2} / m_{b}^{2}\right)$ cancel exactly, leaving $\mathcal{C}_{\rho_{D}}\left(\mu_{1}, m_{b}\right)$ free of any IR divergences. Finally one can solve the renormalisation

\footnotetext{
${ }^{9}$ We do not take into account the spin-orbit operator $\mathcal{O}_{\rho_{L S}}=\bar{b}_{v}\left(i D_{\mu}\right)(i v \cdot D)\left(i D_{\nu}\right)\left(-i \sigma^{\mu \nu}\right) b_{v}$ since its contribution is vanishing within the adopted convention, see eq. (2.23).

${ }^{10}$ In the literature typically colour singlet and colour octet operators are used, see e.g. refs. [3, 6, 7, 56]; however, for our purposes it turns out to be advantageous to use instead the colour singlet and the colour rearranged operators. The trivial transformation between the two bases is given by eq. (2.5).
} 
group equations to run $\mathcal{C}_{\rho_{D}}\left(\mu_{1}, m_{b}\right)$ down to the scale $m_{b} \geq \mu_{0} \gg \Lambda_{\mathrm{QCD}}$ :

$$
\mathcal{C}_{\rho_{D}}\left(\mu_{1}, \mu_{0}\right)=\mathcal{C}_{\rho_{D}}\left(\mu_{1}, m_{b}\right)-\frac{1}{12 \pi^{2}} \log \left(\frac{\mu_{0}^{2}}{m_{b}^{2}}\right) \sum_{q=u, d, s}\left[2 \mathcal{C}_{6,1}^{(q)}\left(\mu_{1}, m_{b}\right)-\mathcal{C}_{6,2}^{(q)}\left(\mu_{1}, m_{b}\right)\right] .
$$

The coefficients $\mathcal{C}_{\rho_{D}}\left(\mu_{1}, \mu_{0}\right)$ are now IR-finite but scale dependent. This $\mu_{0}$-dependence cancels the corresponding one due to the running of the matrix element of the four-quark operators starting already at LO-QCD via the diagram in figure 5:

$$
\left\langle\tilde{\mathcal{O}}_{6, i}^{(q)}\left(\mu_{0}\right)\right\rangle=\left\langle\tilde{\mathcal{O}}_{6, i}^{(q)}\left(m_{b}\right)\right\rangle+\frac{a_{i}}{12 \pi^{2}} \log \left(\frac{\mu_{0}^{2}}{m_{b}^{2}}\right)\left\langle\mathcal{O}_{\rho_{D}}\left(m_{b}\right)\right\rangle .
$$

Our results are presented in the next section. We conclude with the remaining dimensionsix contribution to $\Gamma_{\mathrm{NL}}(B)$ (see appendix $\mathrm{C}$ for the expression of the coefficients at LO-QCD):

$$
\Gamma_{\mathrm{NL}}^{(4 \mathrm{q})}(B)=\frac{\Gamma_{0}}{2 m_{B}} C\left(\mu_{1}\right) \sum_{q=u, d, s} \sum_{i=1}^{4} \mathcal{C}_{6, i}^{(q)}\left(\mu_{1}, \mu_{0}\right) \frac{\left\langle\tilde{\mathcal{O}}_{6, i}^{(q)}\left(\mu_{0}\right)\right\rangle}{m_{b}^{3}},
$$

where the matrix element of the four-quark operators can be parametrised as

$$
\left\langle B_{q^{\prime}}\left|\tilde{\mathcal{O}}_{6, i}^{(q)}\right| B_{q^{\prime}}\right\rangle=A_{i} m_{B}^{2} f_{B}^{2}\left(\mathcal{B}_{i}^{(q)}(B) \delta_{q q^{\prime}}+\tau_{i}^{(q)}(B)\right), \quad q^{\prime}=u, d, s
$$

with $A_{1}=A_{3}=1, A_{2}=A_{4}=\left(m_{B} /\left(m_{b}+m_{q}\right)\right)^{2}$ and $f_{B}$ being the decay constant of the $B$ meson. We have separated the contribution due to the valence and non-valence quarks, where $\mathcal{B}_{i}^{(q)}$ is non vanishing only for $q$ equal to $q^{\prime}$, the spectator quark in the $B$ meson, and is expected to be of order one, see e.g. ref. [56], while $\tau_{i}^{(q)}$ accounts for the effects of an "intrinsic" $q$ quark $[43,44]$ and is expected to be small. Its numerical value can be estimated via e.g. the calculation of the so-called eye contractions in the non-perturbative determination of the matrix elements, see ref. [58].

\section{Results}

The contribution of the Darwin operator to the inclusive non-leptonic decay $b \rightarrow q_{1} \bar{q}_{2} q_{3}$ is presented in the following form

$$
\Gamma_{\mathrm{NL}}^{\left(\rho_{D}\right)}(B)=\Gamma_{0}\left(3 C_{1}^{2} \mathcal{C}_{\rho_{D}, 11}^{\left(q_{1} \bar{q}_{2} q_{3}\right)}+2 C_{1} C_{2} \mathcal{C}_{\rho_{D}, 12}^{\left(q_{1} \bar{q}_{2} q_{3}\right)}+3 C_{2}^{2} \mathcal{C}_{\rho_{D}, 22}^{\left(q_{1} \bar{q}_{2} q_{3}\right)}\right) \frac{\rho_{D}^{3}}{m_{b}^{3}}
$$

where $\rho_{D}^{3}$ is defined in eq. (2.23), $C_{1,2}\left(\mu_{1}\right)$ are the $\Delta B=1$ Wilson coefficients, and

$$
\begin{aligned}
& \mathcal{C}_{\rho_{D}, 11}^{(u \bar{u} d)}=6+8 \log \left(\frac{\mu_{0}^{2}}{m_{b}^{2}}\right), \\
& \mathcal{C}_{\rho_{D}, 12}^{(u \bar{u} d)}=-\frac{34}{3} \\
& \mathcal{C}_{\rho_{D}, 22}^{(u \bar{u} d)}=6+8 \log \left(\frac{\mu_{0}^{2}}{m_{b}^{2}}\right),
\end{aligned}
$$



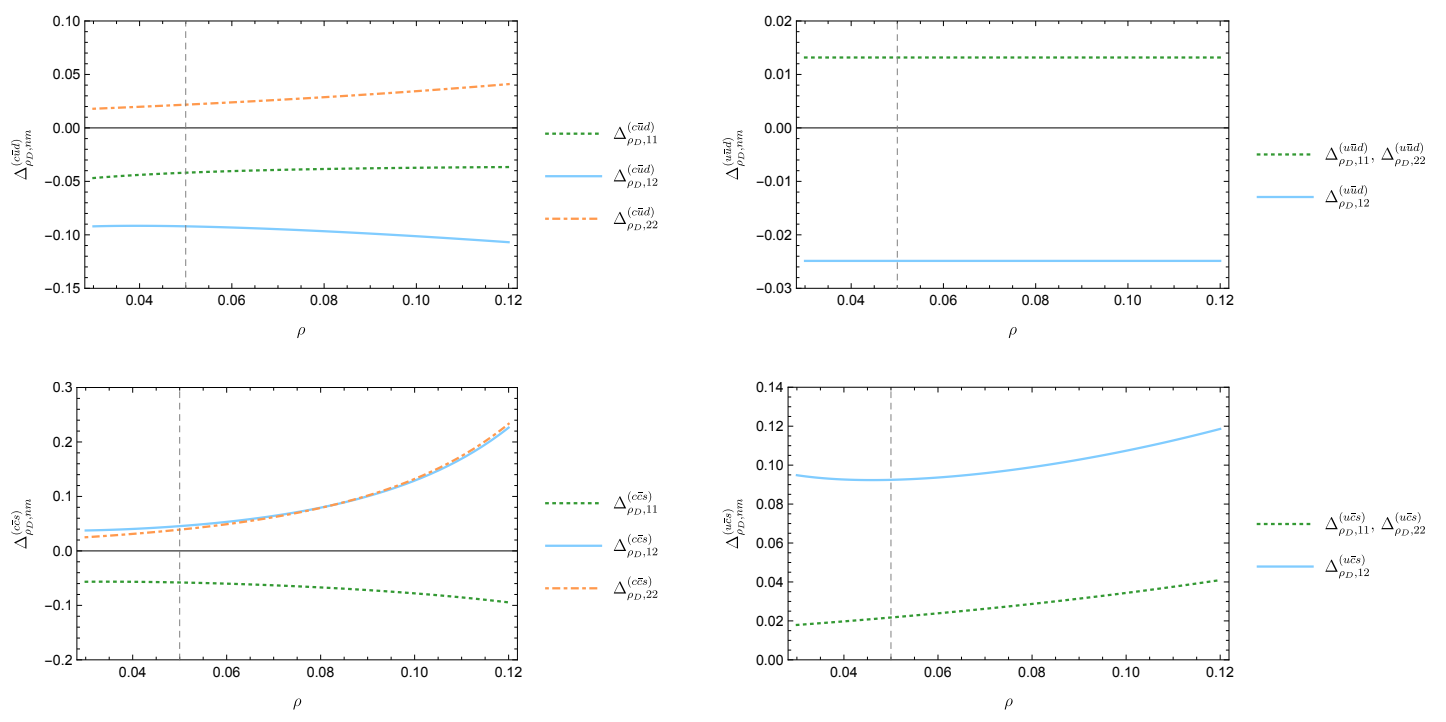

Figure 6. The Darwin term correction normalised to the partonic-level coefficient for different modes, namely $b \rightarrow c \bar{u} d$ (top left), $b \rightarrow u \bar{u} d$ (top right), $b \rightarrow c \bar{c} s$ (bottom left), and $b \rightarrow u \bar{c} s$ (bottom right). The green dotted line corresponds to $Q_{1} \otimes Q_{1}$, the solid cyan line to $Q_{1} \otimes Q_{2}$ and the dotted-dashed orange line to the $Q_{2} \otimes Q_{2}$ contribution. For reference we have fixed the values $\mu_{0}=m_{b}, m_{b}=4.5 \mathrm{GeV}$ and $\rho_{D}^{3}=0.2 \mathrm{GeV}^{3}$. The dashed vertical line shows the approximate value $\rho=0.05$ in the $\overline{\mathrm{MS}}$ scheme.

$$
\begin{aligned}
\mathcal{C}_{\rho_{D}, 11}^{(u \bar{c} s)}= & \frac{2}{3}(1-\rho)\left[9+11 \rho-12 \rho^{2} \log (\rho)-24\left(1-\rho^{2}\right) \log (1-\rho)-25 \rho^{2}+5 \rho^{3}\right] \\
& +8(1-\rho)^{2}(1+\rho) \log \left(\frac{\mu_{0}^{2}}{m_{b}^{2}}\right) \\
\mathcal{C}_{\rho_{D}, 12}^{(u \bar{c} s)}= & \frac{2}{3}\left[-41-12\left(2+5 \rho+2 \rho^{2}-2 \rho^{3}\right) \log (\rho)\right. \\
& \left.\quad-48(1-\rho)^{2}(1+\rho) \log (1-\rho)+26 \rho-18 \rho^{2}+38 \rho^{3}-5 \rho^{4}\right] \\
& +16(1-\rho)^{2}(1+\rho) \log \left(\frac{\mu_{0}^{2}}{m_{b}^{2}}\right) \\
& +8(1-\rho)^{2}(1+\rho) \log \left(\frac{\mu_{0}^{2}}{m_{b}^{2}}\right), \\
\mathcal{C}_{\rho_{D}, 22}^{(u \bar{c} s)}= & \frac{2}{3}(1-\rho)\left[9+11 \rho-12 \rho^{2} \log (\rho)-24\left(1-\rho^{2}\right) \log (1-\rho)-25 \rho^{2}+5 \rho^{3}\right] \\
\mathcal{C}_{\rho_{D}, 11}^{(c \bar{u} d)}= & \frac{2}{3}\left[17+12 \log (\rho)-16 \rho-12 \rho^{2}+16 \rho^{3}-5 \rho^{4}\right] \\
\mathcal{C}_{\rho_{D}, 12}^{(c \bar{u} d)}= & \frac{2}{3}\left[-9+12\left(1-3 \rho^{2}+\rho^{3}\right) \log (\rho)\right. \\
& \left.\quad+24(1-\rho)^{3} \log (1-\rho)+50 \rho-90 \rho^{2}+54 \rho^{3}-5 \rho^{4}\right] \\
& -8(1-\rho)^{3} \log \left(\frac{\mu_{0}^{2}}{m_{b}^{2}}\right)
\end{aligned}
$$




$$
\begin{aligned}
& \mathcal{C}_{\rho_{D}, 22}^{(c \bar{u} d)}=\frac{2}{3}(1-\rho)\left[9+11 \rho-12 \rho^{2} \log (\rho)\right. \\
& \left.-24\left(1-\rho^{2}\right) \log (1-\rho)-25 \rho^{2}+5 \rho^{3}\right] \\
& +8(1-\rho)^{2}(1+\rho) \log \left(\frac{\mu_{0}^{2}}{m_{b}^{2}}\right), \\
& \mathcal{C}_{\rho_{D}, 11}^{(c \bar{c} s)}=\frac{2}{3}\left[\sqrt{1-4 \rho}\left(17+8 \rho-22 \rho^{2}-60 \rho^{3}\right)\right. \\
& \left.-12\left(1-\rho-2 \rho^{2}+2 \rho^{3}+10 \rho^{4}\right) \log \left(\frac{1+\sqrt{1-4 \rho}}{1-\sqrt{1-4 \rho}}\right)\right], \\
& \mathcal{C}_{\rho_{D}, 12}^{(c \bar{c} s)}=\frac{2}{3}\left[\sqrt{1-4 \rho}\left(-45+46 \rho-106 \rho^{2}-60 \rho^{3}\right)\right. \\
& \left.+12\left(1+4 \rho^{2}-16 \rho^{3}-10 \rho^{4}\right) \log \left(\frac{1+\sqrt{1-4 \rho}}{1-\sqrt{1-4 \rho}}\right)\right] \\
& +\left.8\left[\mathcal{M}_{112}(\rho, \eta)-\sqrt{1-4 \rho} \log (\eta)\right]\right|_{\eta \rightarrow 0} \\
& +8 \sqrt{1-4 \rho} \log \left(\frac{\mu_{0}^{2}}{m_{b}^{2}}\right), \\
& \mathcal{C}_{\rho_{D}, 22}^{(c \bar{c} s)}=\frac{2}{3}\left[\sqrt{1-4 \rho}\left(-3+22 \rho-34 \rho^{2}-60 \rho^{3}\right)\right. \\
& \left.-24 \rho\left(1+\rho+2 \rho^{2}+5 \rho^{3}\right) \log \left(\frac{1+\sqrt{1-4 \rho}}{1-\sqrt{1-4 \rho}}\right)\right] \\
& +\left.8\left[\mathcal{M}_{112}(\rho, \eta)-\sqrt{1-4 \rho} \log (\eta)\right]\right|_{\eta \rightarrow 0} \\
& +8 \sqrt{1-4 \rho} \log \left(\frac{\mu_{0}^{2}}{m_{b}^{2}}\right) .
\end{aligned}
$$

The dimensionless parameter $\eta=m_{q}^{2} / m_{b}^{2}$ and the master integral $\mathcal{M}_{112}$ is defined as ${ }^{11}$

$$
\mathcal{M}_{112}(\rho, \eta)=-\int_{(\sqrt{\rho}+\sqrt{\eta})^{2}}^{(1-\sqrt{\rho})^{2}} d t \frac{\left(t^{2}-2(1+\rho) t+(1-\rho)^{2}\right)(t-\eta+\rho)}{t \sqrt{\left(t^{2}-2(1+\rho) t+(1-\rho)^{2}\right)\left(t^{2}-2 t(\eta+\rho)+(\eta-\rho)^{2}\right)}}
$$

Note, that because of $m_{d}=m_{s}=0$ the following relations hold:

$$
\mathcal{C}_{\rho_{D}, n m}^{(c \bar{u} d)}=\mathcal{C}_{\rho_{D}, n m}^{(c \bar{u} s)}, \quad \mathcal{C}_{\rho_{D}, n m}^{(c \bar{c} s)}=\mathcal{C}_{\rho_{D}, n m}^{(c \bar{c} d)}, \quad \mathcal{C}_{\rho_{D}, n m}^{(u \bar{u} d)}=\mathcal{C}_{\rho_{D}, n m}^{(u \bar{u} s)}, \quad \mathcal{C}_{\rho_{D}, n m}^{(u \bar{c} s)}=\mathcal{C}_{\rho_{D}, n m}^{(u \bar{c} d)} .
$$

The relative effect of the Darwin term with respect to the corresponding partonic-level contribution is given by

$$
\Delta_{\rho_{D}, n m}^{\left(q_{1} \bar{q}_{2} q_{3}\right)}=\frac{\mathcal{C}_{\rho_{D}, n m}^{\left(q_{1} \bar{q}_{2} q_{3}\right)}}{\mathcal{C}_{0}^{\left(q_{1} \bar{q}_{2} q_{3}\right)}} \frac{\rho_{D}^{3}}{m_{b}^{3}} .
$$

\footnotetext{
${ }^{11}$ An explicit analytic expression for $\left.\left[\mathcal{M}_{112}(\rho, \eta)-\sqrt{1-4 \rho} \log (\eta)\right]\right|_{\eta \rightarrow 0}$ has been found in ref. [84].
} 


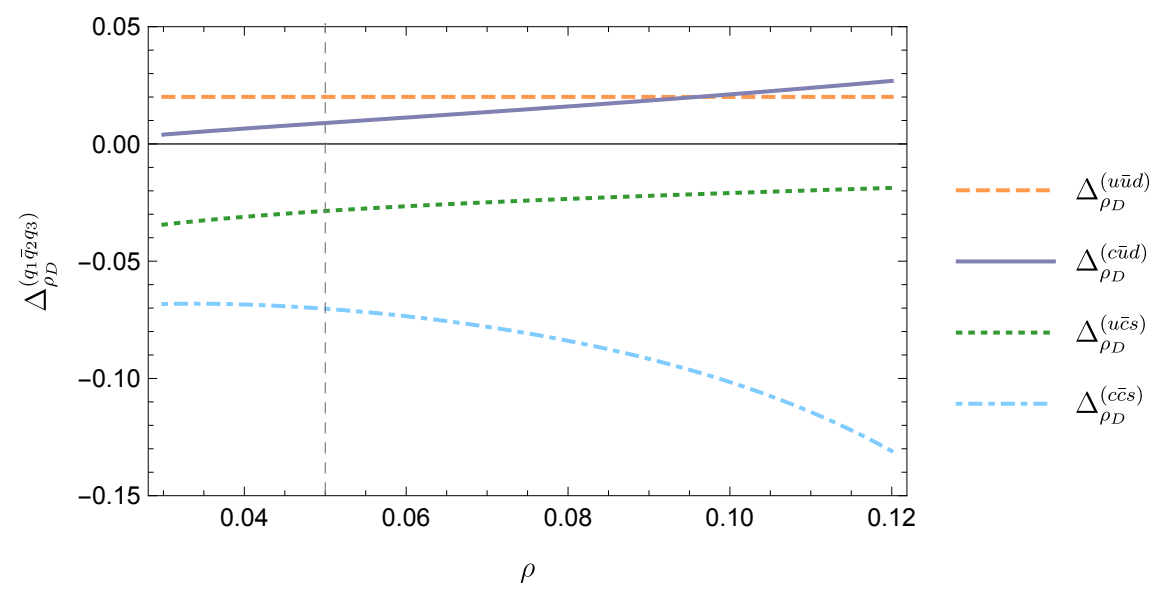

Figure 7. Total relative size of the Darwin term correction compared to the partonic-level contribution for different modes, including $b \rightarrow u \bar{u} d$ (dashed orange), $b \rightarrow c \bar{u} d$ (solid purple), $b \rightarrow u \bar{c} s$ (dotted green) and $b \rightarrow c \bar{c} s$ (dot-dashed cyan). For reference we have fixed the values $\mu_{1}=\mu_{0}=m_{b}$, $m_{b}=4.5 \mathrm{GeV}$ and $\rho_{D}^{3}=0.2 \mathrm{GeV}^{3}$. The dashed vertical line shows the approximate value $\rho=0.05$ in the $\overline{\mathrm{MS}}$ scheme.

In figure 6 , these ratios are plotted as functions of $\rho$ for all the colour structures and the four modes, using for reference the values $\mu_{0}=m_{b}, m_{b}=4.5 \mathrm{GeV}$ and $\rho_{D}^{3}=0.2 \mathrm{GeV}^{3}$. Figure 7 shows the total relative contribution for each mode, namely

$$
\Delta_{\rho_{D}}^{\left(q_{1} \bar{q}_{2} q_{3}\right)}=\frac{3 C_{1}^{2} \mathcal{C}_{\rho_{D}}^{\left(q_{1} \bar{q}_{2} q_{3}\right)}+2 C_{1} C_{2} \mathcal{C}_{\rho_{D}, 12}^{\left(q_{1} \bar{q}_{2} q_{3}\right)}+3 C_{2}^{2} \mathcal{C}_{\rho_{D}, 22}^{\left(q_{1} \bar{q}_{2} q_{3}\right)}}{\left(3 C_{1}^{2}+2 C_{1} C_{2}+3 C_{2}^{2}\right) \mathcal{C}_{0}^{\left(q_{1} \bar{q}_{2} q_{3}\right)}} \frac{\rho_{D}^{3}}{m_{b}^{3}} .
$$

As one can see, the Darwin operator leads to sizeable corrections of the order $1-7 \%$ (for $\rho=0.05)$ to the $b \rightarrow q_{1} \bar{q}_{2} q_{3}$ decay width.

A final comment about the effect of the constant terms $b_{i}$ in eq. (2.32) for the determination of the coefficients $\mathcal{C}_{\rho_{D}, n m}^{\left(q_{1} \bar{q}_{2} q_{3}\right)}$. We have already emphasised that their values depend on the choice of the four-quark operators basis. Consider as an example the coefficient $\mathcal{C}_{\rho_{D}, 12}^{(c \bar{u} d)}$. In our basis given by eqs. (2.30) and $(2.31), \mathcal{C}_{\rho_{D}, 12}^{(c \bar{u} d)}=-29.0$ for the reference value $\rho=0.05$. If instead of eq. (2.30) we had considered the following operators (as it was chosen in ref. [84]):

$$
\tilde{\mathcal{O}}_{6,1}^{(u)}=\left(\bar{b}_{v}^{i} \Gamma^{\sigma} \gamma^{\mu} \Gamma^{\rho} u^{i}\right)\left(\bar{u}^{j} \Gamma_{\sigma} \gamma_{\mu} \Gamma_{\rho} b_{v}^{j}\right), \quad \tilde{\mathcal{O}}_{6,2}^{(u)}=\left(\bar{b}_{v}^{i} \Gamma^{\sigma} \psi \Gamma^{\rho} u^{i}\right)\left(\bar{u}^{j} \Gamma_{\sigma} \psi \Gamma_{\rho} b_{v}^{j}\right),
$$

then in eq. (2.32) we would have obtained $a_{1}=8, b_{1}=-5 / 4$ and $a_{2}=2, b_{2}=-3 / 2$, leading to $\mathcal{C}_{\rho_{D}, 12}^{(c \bar{u} d)}=-24.0$ (for the same value of $\rho$ ). This shift of $\sim 17 \%$ should be compensated (up to higher orders) by the different value of the matrix element of the operators defined in these two bases.

\section{Discussion and conclusion}

This work presents the first determination of the Darwin term contribution to the nonleptonic decay $b \rightarrow q_{1} \bar{q}_{2} q_{3}$. Using the expansion of the quark propagator in the external 


\begin{tabular}{|c|c|c|c|c|c|c|}
\hline & $\mathcal{C}_{G, 11}^{\left(q_{1} \bar{q}_{2} q_{3}\right)}$ & $\mathcal{C}_{G, 12}^{\left(q_{1} \bar{q}_{2} q_{3}\right)}$ & $\mathcal{C}_{G, 22}^{\left(q_{1} \bar{q}_{2} q_{3}\right)}$ & $\mathcal{C}_{\rho_{D}, 11}^{\left(q_{1} \bar{q}_{2} q_{3}\right)}$ & $\mathcal{C}_{\rho_{D}, 12}^{\left(q_{1} \bar{q}_{2} q_{3}\right)}$ & $\mathcal{C}_{\rho_{D}, 22}^{\left(q_{1} \bar{q}_{2} q_{3}\right)}$ \\
\hline$b \rightarrow u \bar{u} d$ & -1.5 & -9.5 & -1.5 & 6 & -11.3 & 6 \\
$b \rightarrow u \bar{c} s$ & -1.28 & -6.23 & -1.28 & 6.84 & 29.1 & 6.84 \\
$b \rightarrow c \bar{u} d$ & -1.28 & -8.14 & -1.28 & -13.2 & -29.0 & 6.84 \\
$b \rightarrow c \bar{c} s$ & -1.05 & -4.99 & -1.05 & -11.5 & -9.02 & 7.74 \\
\hline
\end{tabular}

Table 1. Numerical values of the coefficients $\mathcal{C}_{G, n m}^{\left(q_{1} \bar{q}_{2} q_{3}\right)}$ and $\mathcal{C}_{\rho_{D}, n m}^{\left(q_{1} \bar{q}_{2} q_{3}\right)}$ for $\rho=0.05$ and $\mu_{0}=m_{b}$

gluon field together with the standard technique of the HQE allows to obtain a systematic expansion in inverse powers of the heavy quark mass $m_{b}$. At order $1 / m_{b}^{3}$ operator mixing ensures that the IR divergences arising from the expansion of the light quark propagators cancel, introducing though scale-dependence in the coefficient of $\rho_{D}^{3}$, with the divergent $\log \left(m_{q}^{2} / m_{b}^{2}\right)$ being in fact replaced by $\log \left(\mu_{0}^{2} / m_{b}^{2}\right) .{ }^{12}$ Preliminary numerical analysis reveals that this contribution is sizeable. For illustration purposes, we show the total non-leptonic decay width of the $B^{+}$and $B_{d}$ mesons up to dimension-six and just at LO-QCD. Inputs for the quark masses and the HQE parameters are taken from refs. [46, 56, 58] and we set $\mu_{1}=\mu_{0}=m_{b}$ :

$$
\begin{aligned}
\Gamma_{\mathrm{NL}}(B) & =\Gamma_{3}[1-0.50 \frac{\mu_{\pi}^{2}}{m_{b}^{2}}-0.44 \frac{\mu_{G}^{2}}{m_{b}^{2}}-20.25 \frac{\rho_{D}^{3}}{m_{b}^{3}}+\underbrace{\ldots}_{4 \mathrm{q}-\text { contr. }}] \\
& =\Gamma_{3}[1-\underbrace{0.0112}_{\mu_{\pi}^{2}}-\underbrace{0.0071}_{\mu_{G}^{2}}-\underbrace{0.0373}_{\rho_{D}^{3}}-\underbrace{0.0029}_{\tau_{i}^{(q)}} \underbrace{\left.\begin{array}{c}
-0.1023\left(B^{+}\right) \\
+0.0148\left(B_{d}\right)
\end{array}\right],}_{\mathcal{B}_{i}^{(q)}},
\end{aligned}
$$

where

$$
\Gamma_{3}=\left(3 C_{1}^{2}+2 C_{1} C_{2}+3 C_{2}^{2}\right) \sum_{\left\{q_{1}, q_{2}, q_{3}\right\}} \Gamma_{0}^{\left(q_{1} \bar{q}_{2} q_{3}\right)} \mathcal{C}_{0}^{\left(q_{1} \bar{q}_{2} q_{3}\right)}
$$

and $\Gamma_{0}^{\left(q_{1} \bar{q}_{2} q_{3}\right)}$ is given by eq. (2.25). In eq. (4.1) the effect of the different non-perturbative parameters is shown separately. We find that the new contribution due to the Darwin operator is significant, for the $B_{d}$ meson it is even larger than the dimension-five and the weak-exchange contributions while for the $B^{+}$meson the Pauli interference term still gives the dominant correction. To understand this better, in table 1 we compare the coefficients of the Darwin and of the chromo-magnetic operators ${ }^{13}$ for different modes. As already stated above, at order $1 / m_{b}^{2}$, both diagrams in figure 2 vanish and the coefficient of the kinetic operator as well as $\mathcal{C}_{G, 11}^{\left(q_{1} \bar{q}_{2} q_{3}\right)}$ and $\mathcal{C}_{G, 22}^{\left(q_{1} \bar{q}_{2} q_{3}\right)}$ receive only the small contribution coming from the expansion of the matrix element of dimension-three, cf. eq. (2.15). Hence the dimension-five contribution stemming from the $Q_{1} \otimes Q_{1}$ combination with large $3 C_{1}^{2}$ is actually suppressed. A further pronounced source of cancellation lies in the combination

\footnotetext{
${ }^{12} \mathrm{Up}$ to a finite polynomial in $\rho$, depending on the specific four-quark operator basis adopted.

${ }^{13}$ For simplicity, we do not show the coefficient of $\mu_{\pi}^{2}$ being just $(-1 / 2) \mathcal{C}_{0}^{\left(q_{1} \bar{q}_{2} q_{3}\right)}$ for all three $\Delta B=1$ operator combinations.
} 
$3 C_{1}^{2} \mathcal{C}_{G, 11}^{\left(q_{1} \bar{q}_{2} q_{3}\right)}+2 C_{1} C_{2} \mathcal{C}_{G, 12}^{\left(q_{1} \bar{q}_{2} q_{3}\right)}$ because of the negative value of $C_{2}$ and of the hierarchy $\left|C_{1} / C_{2}\right| \approx \mathcal{C}_{G, 12}^{\left(q_{1} \bar{q}_{2} q_{3}\right)} / \mathcal{C}_{G, 11}^{\left(q_{1} \bar{q}_{2} q_{3}\right)}$. No cancellations are present at order $1 / m_{b}^{3}$ and this explains why, despite the power suppression, the Darwin contribution is larger than the one of dimension-five.

This result does not come as completely new, in fact it was already known in the well studied inclusive semi-leptonic decays, that the Darwin term constitutes a sizeable correction to the decay rate. For example, in the inclusive semil-leptonic $B \rightarrow X_{c} \ell \bar{\nu}_{\ell}$ transition (with $\ell=e, \mu$ ) the following hierarchy (up to $1 / m_{b}^{4}$ and at LO-QCD) is found (see e.g. ref. [62])

$$
\begin{aligned}
\Gamma\left(B \rightarrow X_{c} \ell \bar{\nu}_{\ell}\right) & =\Gamma_{3}^{(c \ell \bar{\nu})}\left[1-0.5 \frac{\mu_{\pi}^{2}}{m_{b}^{2}}-1.9 \frac{\mu_{G}^{2}}{m_{b}^{2}}-19.1 \frac{\rho_{D}^{3}}{m_{b}^{3}}-20.5 \frac{s_{1}}{m_{b}^{4}}\right. \\
& \left.+4.0 \frac{s_{2}}{m_{b}^{4}}-13.5 \frac{s_{3}}{m_{b}^{4}}-9.5 \frac{s_{5}}{m_{b}^{4}}\right] \\
& =\Gamma_{3}^{(c \ell \bar{\nu})}[1-\underbrace{0.043}_{\text {dim-5 }}-\underbrace{0.029}_{\text {dim-6 }}+\underbrace{0.003}_{\operatorname{dim}-7}],
\end{aligned}
$$

where $\Gamma_{3}^{(c \ell \bar{\nu})}=\Gamma_{0}^{(c \ell \bar{\nu})} \mathcal{C}_{0}^{(c \ell \bar{\nu})}$ and the numerical values of parameters are taken from ref. [85].

The size of the coefficients in eq. (4.3) confirms the suppression of the dimension-five contribution but does not seem to point towards an abnormal enhancement of the Darwin operator, whose coefficient is of similar size as the ones of dimension-seven. Note also that the second source of cancellation discussed above is now absent and the dimension-five contribution is larger than in the non-leptonic case. Finally, from eq. (4.3) it does not appear to be any problem with the convergence of the HQE.

To conclude, the inclusion of the Darwin operator contribution could lead to a sizeable modification of the theory prediction for the lifetime ratio $\tau\left(B_{s}\right) / \tau\left(B_{d}\right)$ compared to the high experimental precision, which reaches the accuracy of the four per mille level. An updated theoretical analysis of $\tau\left(B_{s}\right) / \tau\left(B_{d}\right)$ will be performed once the $\mathrm{SU}(3)_{F}$ violation ratio of the Bag parameters and of the matrix elements of the Darwin operator will become available.

\section{Acknowledgments}

We would like to thank Thomas Mannel, Daniel Moreno and Alexei Pivovarov for the very valuable discussions and for sharing their results [84] before publication. These are in perfect agreement with ours and details of the difference in the theoretical methods used can be found in ref. [84]. Moreover we are also grateful to Simon Badger, Ekta Chaubey, Danny King, Robin Marzucca, Thomas Rauh, Johannes Schlenk and Keri Vos for the helpful discussions. This work was supported by the STFC grant of the IPPP.

\section{A Expansion of the quark propagator in the external gluon field}

Following refs. [28, 40], soft gluon interactions of the quark field can be accounted using the background field method. Assuming the $b$ quark embedded in a weakly changing gluon 
field allows to systematically expand the quark propagator as a series in the gluon field strength tensor $G_{\mu \nu}=-i\left[i D_{\mu}, i D_{\nu}\right]$. In the case of a massive quark the expansion of the propagator up to terms linear in $G_{\mu \nu}$ is given in ref. [28], while an expression including terms proportional to $D_{\rho} G_{\mu \nu}=-\left[i D_{\rho},\left[i D_{\mu}, i D_{\nu}\right]\right]$ can be found in ref. [86]. ${ }^{14}$ To compute the propagator, one starts from the Green-function equation,

$$
(i \not \partial+\not A(x)-m) S(x, y)=\delta^{(4)}(x-y),
$$

which admits a solution in form of the perturbative series

$$
i S(x, y)=i S^{(0)}(x-y)+i S^{(1)}(x, y)+\ldots
$$

where $S^{(0)}(x-y)$ is the free-quark propagator and $S^{(1)}(x, y)$ the first order correction:

$$
i S^{(1)}(x, y)=\int d^{4} z i S^{(0)}(x-z) i \not A(z) i S^{(0)}(z-y) .
$$

Using the Fock-Schwinger gauge i.e. $x^{\mu} A_{\mu}(x)=0$, the gluon field is expressible directly in terms of the gluon field strength tensor, see ref. [40] for a detailed derivation:

$$
A_{\mu}^{a}(z)=\int_{0}^{1} d \alpha \alpha z^{\rho} G_{\rho \mu}^{a}(\alpha z) .
$$

Expanding $G_{\rho \mu}(\alpha z)$ around $z=0$ and taking into account that in the Fock-Schwinger gauge $z^{\mu} \partial_{\mu}=z^{\mu} D_{\mu}$, yields:

$$
A_{\mu}^{a}(z)=\frac{1}{2} z^{\rho} G_{\rho \mu}^{a}(0)+\frac{1}{3} z^{\nu} z^{\rho} D_{\nu} G_{\rho \mu}^{a}(0)+\mathcal{O}(D D G)
$$

Substituting the previous expression in eq. (A.3) and setting $y=0$ one obtains

$$
S(x, 0)=\int \frac{d^{4} k}{(2 \pi)^{4}} e^{-i k x} S(k),
$$

where the quark propagator in momentum space reads

$$
\begin{aligned}
S(k)= & \frac{\not k+m}{k^{2}-m^{2}}+\frac{1}{2\left(k^{2}-m^{2}\right)^{2}}\left(-m G_{\rho \mu} \sigma^{\rho \mu}+2 \tilde{G}_{\rho \mu} k^{\rho} \gamma^{\mu} \gamma^{5}\right) \\
& +\frac{2}{3} \frac{D_{\rho} G^{\rho \mu}}{\left(k^{2}-m^{2}\right)^{2}}\left(\gamma_{\mu}-\frac{(k+2 m) k_{\mu}}{k^{2}-m^{2}}\right)-\frac{2}{3} \frac{D_{\nu} G_{\rho \mu} k^{\nu} k^{\rho} \gamma^{\mu}}{\left(k^{2}-m^{2}\right)^{3}} \\
& +\frac{2}{3} \frac{m D_{\nu} G_{\rho \mu}}{\left(k^{2}-m^{2}\right)^{3}}\left(\gamma^{\rho} \gamma^{\mu} k^{\nu}-\gamma^{\mu} \gamma^{\nu} k^{\rho}\right)+2 i \frac{D_{\nu} \tilde{G}_{\rho \mu} k^{\nu} k^{\rho} \gamma^{\mu} \gamma^{5}}{\left(k^{2}-m^{2}\right)^{3}}+\ldots
\end{aligned}
$$

In the above, $\tilde{G}_{\rho \mu}=(1 / 2) \epsilon_{\rho \mu \sigma \eta} G^{\sigma \eta}$ and $\epsilon_{\rho \mu \sigma \eta}$ is the Levi-Civita tensor, while the ellipsis stands for terms with higher derivatives as well as higher powers of $G_{\mu \nu}$. In the limit $m \rightarrow 0$, eq. (A.7) correctly reproduces the massless expression given in ref. [40]. Finally,

\footnotetext{
${ }^{14}$ Note that their expression is not complete as it only contains terms relevant for the computation, namely with odd number of gamma-matrices.
} 
we emphasise that the Fock-Schwinger gauge breaks explicitly the translation invariance of $S(x, y)$, namely:

$$
S(0, x)=\int \frac{d^{4} k}{(2 \pi)^{4}} e^{i k x} \tilde{S}(k), \quad \tilde{S}(k) \neq S(k),
$$

with

$$
\begin{aligned}
\tilde{S}(k)= & \frac{\not k+m}{k^{2}-m^{2}}+\frac{1}{2\left(k^{2}-m^{2}\right)^{2}}\left(-m G_{\rho \mu} \sigma^{\rho \mu}+2 \tilde{G}_{\rho \mu} k^{\rho} \gamma^{\mu} \gamma^{5}\right) \\
& +\frac{2}{3} \frac{D_{\rho} G^{\rho \mu}}{\left(k^{2}-m^{2}\right)^{2}}\left(\gamma_{\mu}-\frac{\not k k_{\mu}}{k^{2}-m^{2}}\right)-\frac{2}{3} \frac{D_{\nu} G_{\rho \mu} k^{\nu} k^{\rho} \gamma^{\mu}}{\left(k^{2}-m^{2}\right)^{3}} \\
& -\frac{2}{3} \frac{m D_{\nu} G_{\rho \mu}}{\left(k^{2}-m^{2}\right)^{3}}\left(\gamma^{\rho} \gamma^{\mu} k^{\nu}-\gamma^{\mu} \gamma^{\nu} k^{\rho}\right)-2 i \frac{D_{\nu} \tilde{G}_{\rho \mu} k^{\nu} k^{\rho} \gamma^{\mu} \gamma^{5}}{\left(k^{2}-m^{2}\right)^{3}}+\ldots
\end{aligned}
$$

For completeness, we also present the equivalent representation of eq. (A.6) in coordinate space

$$
\begin{aligned}
S(x, 0)= & -\frac{i}{4 \pi^{2}} \frac{m^{2} K_{1}\left(m \sqrt{-x^{2}}\right)}{\sqrt{-x^{2}}}-\frac{1}{4 \pi^{2}} \frac{m^{2} \not K_{2}\left(m \sqrt{-x^{2}}\right)}{x^{2}} \\
& -\frac{\tilde{G}_{\alpha \beta}}{8 \pi^{2}} x^{\alpha} \gamma^{\beta} \gamma_{5} \frac{m K_{1}\left(m \sqrt{-x^{2}}\right)}{\sqrt{-x^{2}}}-i \frac{G_{\alpha \beta}}{16 \pi^{2}} \sigma^{\alpha \beta} m K_{0}\left(m \sqrt{-x^{2}}\right) \\
& +\frac{i}{24 \pi^{2}} D_{\alpha} G^{\alpha \beta} \gamma_{\beta} K_{0}\left(m \sqrt{-x^{2}}\right)-\frac{i}{48 \pi^{2}} D_{\alpha} G^{\alpha \beta} x_{\beta} \not \frac{m K_{1}\left(m \sqrt{-x^{2}}\right)}{\sqrt{-x^{2}}} \\
& -\frac{1}{24 \pi^{2}} D_{\alpha} G^{\alpha \beta} x_{\beta} m K_{0}\left(m \sqrt{-x^{2}}\right)-\frac{i}{48 \pi^{2}} D^{\alpha} G^{\beta \rho} \gamma_{\rho} x_{\alpha} x_{\beta} \frac{m K_{1}\left(m \sqrt{-x^{2}}\right)}{\sqrt{-x^{2}}} \\
& -\frac{1}{16 \pi^{2}} D^{\alpha} \tilde{G}^{\beta \rho} \gamma_{\rho} \gamma_{5} x_{\alpha} x_{\beta} \frac{m K_{1}\left(m \sqrt{-x^{2}}\right)}{\sqrt{-x^{2}}}-\frac{1}{48 \pi^{2}} D^{\alpha} G^{\beta \rho} \gamma_{\rho} \gamma_{\beta} x_{\alpha} m K_{0}\left(m \sqrt{-x^{2}}\right) \\
& -\frac{1}{48 \pi^{2}} D^{\alpha} G^{\beta \rho} \gamma_{\rho} \gamma_{\alpha} x_{\beta} m K_{0}\left(m \sqrt{-x^{2}}\right)+\mathcal{O}(D D G),
\end{aligned}
$$

where $K_{0,1,2}(z)$ are the modified Bessel function of the second kind.

\section{B Complementary material to section 2.2}

The divergent coefficients $\mathcal{D}_{n m}^{\left(q_{1} \bar{q}_{2} q_{3}\right)}$ in eq. (2.26) read:

$$
\begin{aligned}
& \mathcal{D}_{11}^{(u \bar{u} d)}=8 \log \left(\frac{m_{u}^{2}}{m_{b}^{2}}\right), \\
& \mathcal{D}_{12}^{(u \bar{u} d)}=8\left[\log \left(\frac{m_{d}^{2}}{m_{b}^{2}}\right)-\log \left(\frac{m_{u}^{2}}{m_{b}^{2}}\right)\right], \\
& \mathcal{D}_{22}^{(u \bar{u} d)}=8 \log \left(\frac{m_{d}^{2}}{m_{b}^{2}}\right), \\
& \mathcal{D}_{11}^{(u \bar{c} s)}=8(1-\rho)^{2}(1+\rho) \log \left(\frac{m_{u}^{2}}{m_{b}^{2}}\right),
\end{aligned}
$$




$$
\begin{aligned}
& \mathcal{D}_{12}^{(u \bar{c} s)}=8(1-\rho)^{2}(1+\rho)\left[\log \left(\frac{m_{u}^{2}}{m_{b}^{2}}\right)+\log \left(\frac{m_{s}^{2}}{m_{b}^{2}}\right)\right], \\
& \mathcal{D}_{22}^{(u \bar{c} s)}=8(1-\rho)^{2}(1+\rho) \log \left(\frac{m_{s}^{2}}{m_{b}^{2}}\right), \\
& \mathcal{D}_{12}^{(c \bar{u} d)}=-16(1-\rho)^{2} \log \left(\frac{m_{u}^{2}}{m_{b}^{2}}\right)+8(1-\rho)^{2}(1+\rho) \log \left(\frac{m_{d}^{2}}{m_{b}^{2}}\right), \\
& \mathcal{D}_{22}^{(c \bar{u} d)}=8(1-\rho)^{2}(1+\rho) \log \left(\frac{m_{d}^{2}}{m_{b}^{2}}\right), \\
& \mathcal{D}_{12}^{(c \bar{c} s)}=8 \sqrt{1-4 \rho} \log \left(\frac{m_{s}^{2}}{m_{b}^{2}}\right), \\
& \mathcal{D}_{22}^{(c \bar{c} s)}=8 \sqrt{1-4 \rho} \log \left(\frac{m_{s}^{2}}{m_{b}^{2}}\right) .
\end{aligned}
$$

The discontinuity of the WA, PI and WE diagrams in figure 4 at LO-QCD, respectively is

$$
\begin{aligned}
\Gamma^{\mathrm{WA}}= & \frac{\Gamma_{0}}{2 m_{B}} \frac{16 \pi^{2}}{m_{b}^{3}} \sqrt{\lambda\left(1, z_{2}, z_{3}\right)}\left\{\left(N_{c} C_{1}^{2}+2 C_{1} C_{2}\right)\right. \\
& \times\left[\left(\left(z_{2}-z_{3}\right)^{2}+z_{2}+z_{3}-2\right) \tilde{\mathcal{O}}_{6,1}^{\left(q_{1}\right)}\right. \\
& \left.-2\left(2\left(z_{2}-z_{3}\right)^{2}-1-z_{2}-z_{3}\right) \tilde{\mathcal{O}}_{6,2}^{\left(q_{1}\right)}\right] \\
& +C_{2}^{2}\left[\left(\left(z_{2}-z_{3}\right)^{2}+z_{2}+z_{3}-2\right) \tilde{\mathcal{O}}_{6,3}^{\left(q_{1}\right)}\right. \\
\Gamma^{\mathrm{PI}}= & \frac{\Gamma_{0}}{2 m_{B}} \frac{96 \pi^{2}}{m_{b}^{3}} \sqrt{\lambda\left(1, z_{1}, z_{3}\right)}\left(1-z_{1}-z_{3}\right) \\
& \times\left[2 C_{1} C_{2} \tilde{\mathcal{O}}_{6,1}^{\left(q_{2}\right)}+\left(z_{1}^{2}+C_{2}^{2}\right) \tilde{\mathcal{O}}_{6,3}^{\left(q_{2}\right)}\right] \\
\Gamma^{\mathrm{WE}}= & \frac{\Gamma_{0}}{2 m_{B}} \frac{16 \pi^{2}}{m_{b}^{3}} \sqrt{\lambda\left(1, z_{1}, z_{2}\right)}\left\{\left(2 C_{1} C_{2}+z_{2}\right)_{3} C_{2}^{2}\right) \\
& \left.\times\left[\left(\left(z_{1}-z_{2}\right)^{2}+z_{1}+z_{2}-2\right)\right]\right\} \\
& +C_{1}^{2}\left[\left(\left(z_{1}-\tilde{\mathcal{O}}_{6,1}^{\left(q_{3}\right)}\right.\right.\right.
\end{aligned}
$$


where $z_{i}=m_{q_{i}}^{2} / m_{b}^{2}$ and the four-quark operators $\tilde{\mathcal{O}}_{6, i}^{(q)}$ are defined in eqs. (2.30), (2.31). Note that, since we set $m_{u}=m_{d}=m_{s}=0$, in our case $z_{i}$ can be either equal to $\rho$ or 0 .

\section{Contribution of four-quark operators at order $1 / m_{b}^{3}$}

The four-quark operators contribution to the non-leptonic decay $b \rightarrow q_{1} \bar{q}_{2} q_{3}$ at order $1 / m_{b}^{3}$ and at LO-QCD is written in the following form

$$
\Gamma_{\mathrm{NL}}^{(4 \mathrm{q})}(B)=\frac{\Gamma_{0}}{2 m_{B}}\left(3 C_{1}^{2} \mathcal{P}_{11}^{\left(q_{1} \bar{q}_{2} q_{3}\right)}+2 C_{1} C_{2} \mathcal{P}_{12}^{\left(q_{1} \bar{q}_{2} q_{3}\right)}+3 C_{2}^{2} \mathcal{P}_{22}^{\left(q_{1} \bar{q}_{2} q_{3}\right)}\right)
$$

where

$$
\begin{aligned}
& P_{11}^{(u \bar{u} d)}=\frac{32 \pi^{2}}{m_{b}^{3}}\left[-\left\langle\tilde{\mathcal{O}}_{6,1}^{(u)}\right\rangle+\left\langle\tilde{\mathcal{O}}_{6,2}^{(u)}\right\rangle+\left\langle\tilde{\mathcal{O}}_{6,3}^{(u)}\right\rangle-\frac{\left\langle\tilde{\mathcal{O}}_{6,3}^{(d)}\right\rangle-\left\langle\tilde{\mathcal{O}}_{6,4}^{(d)}\right\rangle}{3}\right], \\
& P_{12}^{(u \bar{u} d)}=\frac{32 \pi^{2}}{m_{b}^{3}}\left[2\left\langle\tilde{\mathcal{O}}_{6,1}^{(u)}\right\rangle+\left\langle\tilde{\mathcal{O}}_{6,2}^{(u)}\right\rangle-\left\langle\tilde{\mathcal{O}}_{6,1}^{(d)}\right\rangle+\left\langle\tilde{\mathcal{O}}_{6,2}^{(d)}\right\rangle\right], \\
& P_{22}^{(u \bar{u} d)}=\frac{32 \pi^{2}}{m_{b}^{3}}\left[-\left\langle\tilde{\mathcal{O}}_{6,1}^{(d)}\right\rangle+\left\langle\tilde{\mathcal{O}}_{6,2}^{(d)}\right\rangle+\frac{2\left\langle\tilde{\mathcal{O}}_{6,3}^{(u)}\right\rangle+\left\langle\tilde{\mathcal{O}}_{6,4}^{(u)}\right\rangle}{3}\right], \\
& P_{11}^{(u \bar{c} s)}=\frac{32 \pi^{2}}{m_{b}^{3}}(1-\rho)^{2}\left[(1+2 \rho)\left(\left\langle\tilde{\mathcal{O}}_{6,2}^{(u)}\right\rangle+\frac{1}{3}\left\langle\tilde{\mathcal{O}}_{6,4}^{(s)}\right\rangle\right)-\left(1+\frac{\rho}{2}\right)\left(\left\langle\tilde{\mathcal{O}}_{6,1}^{(u)}\right\rangle+\frac{1}{3}\left\langle\tilde{\mathcal{O}}_{6,3}^{(s)}\right\rangle\right)\right], \\
& P_{12}^{(u \bar{c} s)}=\frac{32 \pi^{2}}{m_{b}^{3}}(1-\rho)^{2}\left[(1+2 \rho)\left(\left\langle\tilde{\mathcal{O}}_{6,2}^{(u)}\right\rangle+\left\langle\tilde{\mathcal{O}}_{6.2}^{(s)}\right\rangle\right)-\left(1+\frac{\rho}{2}\right)\left(\left\langle\tilde{\mathcal{O}}_{6,1}^{(u)}\right\rangle+\left\langle\tilde{\mathcal{O}}_{6,1}^{(s)}\right\rangle\right)\right], \\
& P_{22}^{(u \bar{c} s)}=\frac{32 \pi^{2}}{m_{b}^{3}}(1-\rho)^{2}\left[(1+2 \rho)\left(\left\langle\tilde{\mathcal{O}}_{6,2}^{(s)}\right\rangle+\frac{1}{3}\left\langle\tilde{\mathcal{O}}_{6,4}^{(u)}\right\rangle\right)-\left(1+\frac{\rho}{2}\right)\left(\left\langle\tilde{\mathcal{O}}_{6,1}^{(s)}\right\rangle+\frac{1}{3}\left\langle\tilde{\mathcal{O}}_{6,3}^{(u)}\right\rangle\right)\right], \\
& P_{11}^{(c \bar{u} d)}=\frac{32 \pi^{2}}{3 m_{b}^{3}}(1-\rho)^{2}\left[(1+2 \rho)\left\langle\tilde{\mathcal{O}}_{6,4}^{(d)}\right\rangle-\left(1+\frac{\rho}{2}\right)\left\langle\tilde{\mathcal{O}}_{6,3}^{(d)}\right\rangle+3\left\langle\tilde{\mathcal{O}}_{6,3}^{(u)}\right\rangle\right] \\
& P_{12}^{(c \bar{u} d)}=\frac{32 \pi^{2}}{m_{b}^{3}}(1-\rho)^{2}\left[(1+2 \rho)\left\langle\tilde{\mathcal{O}}_{6,2}^{(d)}\right\rangle-\left(1+\frac{\rho}{2}\right)\left\langle\tilde{\mathcal{O}}_{6,1}^{(d)}\right\rangle+3\left\langle\tilde{\mathcal{O}}_{6,1}^{(u)}\right\rangle\right], \\
& P_{22}^{(c \bar{u} d)}=\frac{32 \pi^{2}}{m_{b}^{3}}(1-\rho)^{2}\left[(1+2 \rho)\left\langle\tilde{\mathcal{O}}_{6,2}^{(d)}\right\rangle-\left(1+\frac{\rho}{2}\right)\left\langle\tilde{\mathcal{O}}_{6,1}^{(d)}\right\rangle+\left\langle\tilde{\mathcal{O}}_{6,3}^{(u)}\right\rangle\right], \\
& P_{11}^{(c \bar{c} s)}=\frac{32 \pi^{2}}{3 m_{b}^{3}} \sqrt{1-4 \rho}\left[(1+2 \rho)\left\langle\tilde{\mathcal{O}}_{6,4}^{(s)}\right\rangle-(1-\rho)\left\langle\tilde{\mathcal{O}}_{6,3}^{(s)}\right\rangle\right] \\
& P_{12}^{(c \bar{c} s)}=\frac{32 \pi^{2}}{m_{b}^{3}} \sqrt{1-4 \rho}\left[(1+2 \rho)\left\langle\tilde{\mathcal{O}}_{6,2}^{(s)}\right\rangle-(1-\rho)\left\langle\tilde{\mathcal{O}}_{6,1}^{(s)}\right\rangle\right], \\
& P_{22}^{(c \bar{c} s)}=\frac{32 \pi^{2}}{m_{b}^{3}} \sqrt{1-4 \rho}\left[(1+2 \rho)\left\langle\tilde{\mathcal{O}}_{6,2}^{(s)}\right\rangle-(1-\rho)\left\langle\tilde{\mathcal{O}}_{6,1}^{(s)}\right\rangle\right] .
\end{aligned}
$$

The corresponding expressions for $\mathcal{P}_{n m}^{(u \bar{u} s)}, \mathcal{P}_{n m}^{(u \bar{c} d)}, \mathcal{P}_{n m}^{(c \bar{u} s)}, \mathcal{P}_{n m}^{(c \bar{c} d)}$ can be obtained from the above ones by replacing $\tilde{\mathcal{O}}_{6, i}^{(d)} \leftrightarrow \tilde{\mathcal{O}}_{6, i}^{(s)}$. 


\section{Coefficients of the dimension-three and of the chromo-magnetic op- erators}

Here we present the analytic expressions for the coefficients of the dimension-three and chromo-magnetic operators intoduced in eq. (2.24). They read respectively

$$
\begin{aligned}
& \mathcal{C}_{0}^{(u \bar{u} d)}=1, \quad \mathcal{C}_{G, 11}^{(u \bar{u} d)}=\mathcal{C}_{G, 22}^{(u \bar{u} d)}=-\frac{3}{2}, \quad \mathcal{C}_{G, 12}^{(u \bar{u} d)}=-\frac{19}{2}, \\
& \mathcal{C}_{0}^{(u \bar{c} s)}=1-8 \rho-12 \rho^{2} \log (\rho)+8 \rho^{3}-\rho^{4}, \\
& \mathcal{C}_{G, 11}^{(u \bar{c} s)}=\mathcal{C}_{G, 22}^{(u \bar{c} s)}=-\frac{1}{2}\left(3-8 \rho+12 \rho^{2} \log (\rho)+24 \rho^{2}-24 \rho^{3}+5 \rho^{4}\right), \\
& \mathcal{C}_{G, 12}^{(u \bar{c} s)}=-\frac{1}{2}\left(19+16 \rho+12 \rho(\rho+4) \log (\rho)-24 \rho^{2}-16 \rho^{3}+5 \rho^{4}\right), \\
& \mathcal{C}_{0}^{(c \bar{u} d)}=1-8 \rho-12 \rho^{2} \log (\rho)+8 \rho^{3}-\rho^{4}, \\
& \mathcal{C}_{G, 11}^{(c \bar{u} d)}=\mathcal{C}_{G, 22}^{(c \bar{u} d)}=-\frac{1}{2}\left(3-8 \rho+12 \rho^{2} \log (\rho)+24 \rho^{2}-24 \rho^{3}+5 \rho^{4}\right), \\
& \mathcal{C}_{G, 12}^{(c \bar{u} d)}=-\frac{1}{2}\left(19-56 \rho+12 \rho^{2} \log (\rho)+72 \rho^{2}-40 \rho^{3}+5 \rho^{4}\right), \\
& \mathcal{C}_{0}^{(c \bar{c} s)}=\sqrt{1-4 \rho}\left(1-14 \rho-2 \rho^{2}-12 \rho^{3}\right)+24 \rho^{2}\left(1-\rho^{2}\right) \log \left(\frac{1+\sqrt{1-4 \rho}}{1-\sqrt{1-4 \rho}}\right) \\
& \mathcal{C}_{G, 11}^{(c \bar{c} s)}=\mathcal{C}_{G, 22}^{(c \bar{c} s)}=-\frac{1}{2}\left[\sqrt{1-4 \rho}\left(3-10 \rho+10 \rho^{2}+60 \rho^{3}\right)\right. \\
& \left.-24 \rho^{2}\left(1-5 \rho^{2}\right) \log \left(\frac{1+\sqrt{1-4 \rho}}{1-\sqrt{1-4 \rho}}\right)\right], \\
& \mathcal{C}_{G, 12}^{(c \bar{c} s)}=-\frac{1}{2}\left[\sqrt{1-4 \rho}\left(19-2 \rho+58 \rho^{2}+60 \rho^{3}\right)\right. \\
& \left.-24 \rho\left(2+\rho-4 \rho^{2}-5 \rho^{3}\right) \log \left(\frac{1+\sqrt{1-4 \rho}}{1-\sqrt{1-4 \rho}}\right)\right] .
\end{aligned}
$$

Open Access. This article is distributed under the terms of the Creative Commons Attribution License (CC-BY 4.0), which permits any use, distribution and reproduction in any medium, provided the original author(s) and source are credited.

\section{References}

[1] V.A. Khoze and M.A. Shifman, Heavy quarks, Sov. Phys. Usp. 26 (1983) 387 [inSPIRE].

[2] M.A. Shifman and M.B. Voloshin, Hierarchy of lifetimes of charmed and beautiful hadrons, Sov. Phys. JETP 64 (1986) 698 [Zh. Eksp. Teor. Fiz. 91 (1986) 1180].

[3] A. Lenz, Lifetimes and heavy quark expansion, Int. J. Mod. Phys. A 30 (2015) 1543005 [arXiv: 1405.3601] [INSPIRE]. 
[4] HFLAV collaboration, Averages of b-hadron, c-hadron, and $\tau$-lepton properties as of 2018 , arXiv:1909.12524 [INSPIRE].

[5] G. Buchalla, A.J. Buras and M.E. Lautenbacher, Weak decays beyond leading logarithms, Rev. Mod. Phys. 68 (1996) 1125 [hep-ph/9512380] [INSPIRE].

[6] N.G. Uraltsev, On the problem of boosting nonleptonic b baryon decays, Phys. Lett. B 376 (1996) 303 [hep-ph/9602324] [INSPIRE].

[7] M. Neubert and C.T. Sachrajda, Spectator effects in inclusive decays of beauty hadrons, Nucl. Phys. B 483 (1997) 339 [hep-ph/9603202] [INSPIRE].

[8] Q. Ho-kim and X.Y. Pham, Exact one gluon corrections for inclusive weak processes, Ann. Phys. 155 (1984) 202.

[9] G. Altarelli and S. Petrarca, Inclusive beauty decays and the spectator model, Phys. Lett. B 261 (1991) 303.

[10] M.B. Voloshin, QCD radiative enhancement of the decay $b \rightarrow c \bar{c} s$, Phys. Rev. D 51 (1995) 3948 [hep-ph/9409391] [INSPIRE].

[11] E. Bagan, P. Ball, V.M. Braun and P. Gosdzinsky, Charm quark mass dependence of QCD corrections to nonleptonic inclusive B decays, Nucl. Phys. B 432 (1994) 3 [hep-ph/9408306] [INSPIRE].

[12] E. Bagan, P. Ball, B. Fiol and P. Gosdzinsky, Next-to-leading order radiative corrections to the decay $b \rightarrow c c s$, Phys. Lett. B 351 (1995) 546 [hep-ph/9502338] [INSPIRE].

[13] A. Lenz, U. Nierste and G. Ostermaier, Penguin diagrams, charmless B decays and the missing charm puzzle, Phys. Rev. D 56 (1997) 7228 [hep-ph/9706501] [InSPIRE].

[14] A. Lenz, U. Nierste and G. Ostermaier, Determination of the CKM angle gamma and $\left|V_{u b} / V_{c b}\right|$ from inclusive direct $C P$ asymmetries and branching ratios in charmless $B$ decays, Phys. Rev. D 59 (1999) 034008 [hep-ph/9802202] [InSPIRE].

[15] F. Krinner, A. Lenz and T. Rauh, The inclusive decay $b \rightarrow c \bar{c} s$ revisited, Nucl. Phys. B 876 (2013) 31 [arXiv:1305.5390] [INSPIRE].

[16] A. Czarnecki and K. Melnikov, Two loop QCD corrections to semileptonic b decays at maximal recoil, Phys. Rev. Lett. 78 (1997) 3630 [hep-ph/9703291] [INSPIRE].

[17] A. Czarnecki and K. Melnikov, Two-loop QCD corrections to semileptonic $b$ decays at an intermediate recoil, Phys. Rev. D 59 (1999) 014036 [hep-ph/9804215] [INSPIRE].

[18] T. van Ritbergen, The second order QCD contribution to the semileptonic $b \rightarrow u$ decay rate, Phys. Lett. B 454 (1999) 353 [hep-ph/9903226] [INSPIRE].

[19] K. Melnikov, $O\left(\alpha_{s}^{2}\right)$ corrections to semileptonic decay $b \rightarrow$ cl $\bar{\nu}_{l}$, Phys. Lett. B 666 (2008) 336 [arXiv: 0803.0951] [INSPIRE].

[20] A. Pak and A. Czarnecki, Heavy-to-heavy quark decays at NNLO, Phys. Rev. D 78 (2008) 114015 [arXiv:0808.3509] [INSPIRE].

[21] A. Pak and A. Czarnecki, Mass effects in muon and semileptonic $b \rightarrow c$ decays, Phys. Rev. Lett. 100 (2008) 241807 [arXiv: 0803.0960] [INSPIRE].

[22] M. Dowling, A. Pak and A. Czarnecki, Semi-leptonic b-decay at intermediate recoil, Phys. Rev. D 78 (2008) 074029 [arXiv:0809.0491] [INSPIRE]. 
[23] R. Bonciani and A. Ferroglia, Two-loop QCD corrections to the heavy-to-light quark decay, JHEP 11 (2008) 065 [arXiv: 0809.4687] [INSPIRE].

[24] S. Biswas and K. Melnikov, Second order QCD corrections to inclusive semileptonic $b \rightarrow X_{c} l \bar{\nu}_{l}$ decays with massless and massive lepton, JHEP 02 (2010) 089 [arXiv:0911.4142] [INSPIRE].

[25] M. Brucherseifer, F. Caola and K. Melnikov, On the $O\left(\alpha_{s}^{2}\right)$ corrections to $b \rightarrow X_{u} e \bar{\nu}$ inclusive decays, Phys. Lett. B $\mathbf{7 2 1}$ (2013) 107 [arXiv:1302.0444] [INSPIRE].

[26] A. Czarnecki, M. Ślusarczyk and F.V. Tkachov, Enhancement of the hadronic b quark decays, Phys. Rev. Lett. 96 (2006) 171803 [hep-ph/0511004] [INSPIRE].

[27] I.I.Y. Bigi, N.G. Uraltsev and A.I. Vainshtein, Nonperturbative corrections to inclusive beauty and charm decays: QCD versus phenomenological models, Phys. Lett. B 293 (1992) 430 [Erratum ibid. 297 (1992) 477] [hep-ph/9207214] [INSPIRE].

[28] B. Blok and M.A. Shifman, The rule of discarding $1 / N_{c}$ in inclusive weak decays. 1 , Nucl. Phys. B 399 (1993) 441 [hep-ph/9207236] [InSPIRE].

[29] B. Blok and M.A. Shifman, The rule of discarding $1 / N_{c}$ in inclusive weak decays. 2, Nucl. Phys. B 399 (1993) 459 [hep-ph/9209289] [INSPIRE].

[30] I.I.Y. Bigi, B. Blok, M.A. Shifman, N.G. Uraltsev and A.I. Vainshtein, A QCD 'manifesto' on inclusive decays of beauty and charm, in the proceedings of the $7^{\text {th }}$ Meeting of the APS Division of Particles Fields, November 10-14, Batavia, U.S.A. (1992), hep-ph/9212227 [INSPIRE].

[31] A. Alberti, P. Gambino and S. Nandi, Perturbative corrections to power suppressed effects in semileptonic B decays, JHEP 01 (2014) 147 [arXiv:1311.7381] [INSPIRE].

[32] T. Mannel, A.A. Pivovarov and D. Rosenthal, Inclusive semileptonic B decays from QCD with NLO accuracy for power suppressed terms, Phys. Lett. B 741 (2015) 290 [arXiv: 1405.5072] [INSPIRE].

[33] T. Mannel, A.A. Pivovarov and D. Rosenthal, Inclusive weak decays of heavy hadrons with power suppressed terms at NLO, Phys. Rev. D 92 (2015) 054025 [arXiv:1506.08167] [INSPIRE].

[34] M. Gremm and A. Kapustin, Order $1 / m_{b}^{3}$ corrections to $B \rightarrow X_{c}$ lepton anti-neutrino decay and their implication for the measurement of $\bar{\Lambda}$ and $\lambda_{1}$, Phys. Rev. D $5 \mathbf{5}$ (1997) 6924 [hep-ph/9603448] [INSPIRE].

[35] T. Mannel and A.A. Pivovarov, QCD corrections to inclusive heavy hadron weak decays at $\Lambda_{\mathrm{QCD}}^{3} / m_{Q}^{3}$, Phys. Rev. D 100 (2019) 093001 [arXiv: 1907.09187] [INSPIRE].

[36] M. Beneke, G. Buchalla, C. Greub, A. Lenz and U. Nierste, The $B^{+}-B_{d}^{0}$ lifetime difference beyond leading logarithms, Nucl. Phys. B 639 (2002) 389 [hep-ph/0202106] [INSPIRE].

[37] E. Franco, V. Lubicz, F. Mescia and C. Tarantino, Lifetime ratios of beauty hadrons at the next-to-leading order in QCD, Nucl. Phys. B 633 (2002) 212 [hep-ph/0203089] [InSPIRE].

[38] A. Lenz and T. Rauh, D-meson lifetimes within the heavy quark expansion, Phys. Rev. D 88 (2013) 034004 [arXiv: 1305. 3588] [INSPIRE].

[39] F. Gabbiani, A.I. Onishchenko and A.A. Petrov, Spectator effects and lifetimes of heavy hadrons, Phys. Rev. D 70 (2004) 094031 [hep-ph/0407004] [INSPIRE]. 
[40] V.A. Novikov, M.A. Shifman, A.I. Vainshtein and V.I. Zakharov, Calculations in external fields in quantum chromodynamics. Technical review, Fortsch. Phys. 32 (1984) 585.

[41] D. Benson, I.I. Bigi, T. Mannel and N. Uraltsev, Imprecated, yet impeccable: on the theoretical evaluation of $\Gamma\left(B \rightarrow X_{c} l \nu\right)$, Nucl. Phys. B 665 (2003) 367 [hep-ph/0302262] [INSPIRE].

[42] I.I. Bigi, N. Uraltsev and R. Zwicky, On the nonperturbative charm effects in inclusive $B \rightarrow X_{c} l \nu$ decays, Eur. Phys. J. C 50 (2007) 539 [hep-ph/0511158] [INSPIRE].

[43] M. Fael, T. Mannel and K.K. Vos, The heavy quark expansion for inclusive semileptonic charm decays revisited, JHEP 12 (2019) 067 [arXiv:1910.05234] [INSPIRE].

[44] C. Breidenbach, T. Feldmann, T. Mannel and S. Turczyk, On the role of 'intrinsic charm' in semi-leptonic B-meson decays, Phys. Rev. D 78 (2008) 014022 [arXiv:0805.0971] [InSPIRE].

[45] I. Bigi, T. Mannel, S. Turczyk and N. Uraltsev, The two roads to 'intrinsic charm' in B decays, JHEP 04 (2010) 073 [arXiv:0911.3322] [InSPIRE].

[46] A. Alberti, P. Gambino, K.J. Healey and S. Nandi, Precision determination of the Cabibbo-Kobayashi-Maskawa element $V_{c b}$, Phys. Rev. Lett. 114 (2015) 061802 [arXiv:1411.6560] [INSPIRE].

[47] Fermilab Lattice, MILC, TUMQCD collaboration, Up-, down-, strange-, charm-, and bottom-quark masses from four-flavor lattice QCD, Phys. Rev. D 98 (2018) 054517 [arXiv: 1802.04248] [INSPIRE].

[48] P. Gambino, A. Melis and S. Simula, Extraction of heavy-quark-expansion parameters from unquenched lattice data on pseudoscalar and vector heavy-light meson masses, Phys. Rev. D 96 (2017) 014511 [arXiv: 1704.06105] [INSPIRE].

[49] JLQCD collaboration, Heavy quark expansion parameters from lattice NRQCD, Phys. Rev. D 69 (2004) 094512 [hep-lat/0305024] [INSPIRE].

[50] A.S. Kronfeld and J.N. Simone, Computation of $\bar{\Lambda}$ and $\lambda_{1}$ with lattice QCD, Phys. Lett. $B$ 490 (2000) 228 [Erratum ibid. 495 (2000) 441] [hep-ph/0006345] [INSPIRE].

[51] V. Giménez, G. Martinelli and C.T. Sachrajda, A high statistics lattice calculation of $\lambda_{1}$ and $\lambda_{2}$ in the B meson, Nucl. Phys. B 486 (1997) 227 [hep-lat/9607055] [INSPIRE].

[52] P. Ball and V.M. Braun, Next-to-leading order corrections to meson masses in the heavy quark effective theory, Phys. Rev. D 49 (1994) 2472 [hep-ph/9307291] [INSPIRE].

[53] I.I.Y. Bigi, M.A. Shifman, N.G. Uraltsev and A.I. Vainshtein, Sum rules for heavy flavor transitions in the SV limit, Phys. Rev. D 52 (1995) 196 [hep-ph/9405410] [InSPIRE].

[54] M. Neubert, QCD sum rule calculation of the kinetic energy and chromo interaction of heavy quarks inside mesons, Phys. Lett. B 389 (1996) 727 [hep-ph/9608211] [INSPIRE].

[55] I.I. Bigi, T. Mannel and N. Uraltsev, Semileptonic width ratios among beauty hadrons, JHEP 09 (2011) 012 [arXiv: 1105.4574] [INSPIRE].

[56] M. Kirk, A. Lenz and T. Rauh, Dimension-six matrix elements for meson mixing and lifetimes from sum rules, JHEP 12 (2017) 068 [Erratum ibid. 06 (2020) 162] [arXiv: 1711.02100] [INSPIRE].

[57] D. King, A. Lenz and T. Rauh, $B_{s}$ mixing observables and $\left|V_{t d} / V_{t s}\right|$ from sum rules, JHEP 05 (2019) 034 [arXiv: 1904.00940] [INSPIRE].

[58] D. King, A. Lenz and T. Rauh, in preparation. 
[59] M. Beneke, G. Buchalla and I. Dunietz, Width difference in the $B_{s}-\bar{B}_{s}$ system, Phys. Rev. D 54 (1996) 4419 [Erratum ibid. 83 (2011) 119902] [hep-ph/9605259] [INSPIRE].

[60] Y.-Y. Keum and U. Nierste, Probing penguin coefficients with the lifetime ratio tau $\left(B_{s}\right) / \tau\left(B_{d}\right)$, Phys. Rev. D 57 (1998) 4282 [hep-ph/9710512] [INSPIRE].

[61] A. Lenz and U. Nierste, Numerical updates of lifetimes and mixing parameters of B mesons, in the proceedings of the $6^{\text {th }}$ International Workshop on the CKM Unitarity Triangle (CKM 2010), September 6-10, Warwick, U.K. (2010), arXiv:1102.4274 [INSPIRE].

[62] B.M. Dassinger, T. Mannel and S. Turczyk, Inclusive semi-leptonic B decays to order $1 / m_{b}^{4}$, JHEP 03 (2007) 087 [hep-ph/0611168] [INSPIRE].

[63] T. Mannel, S. Turczyk and N. Uraltsev, Higher order power corrections in inclusive $B$ decays, JHEP 11 (2010) 109 [arXiv:1009.4622] [INSPIRE].

[64] T. Mannel, A.V. Rusov and F. Shahriaran, Inclusive semitauonic B decays to order $\mathcal{O}\left(\Lambda_{\mathrm{QCD}}^{3} / m_{b}^{3}\right)$, Nucl. Phys. B 921 (2017) 211 [arXiv: 1702.01089] [INSPIRE].

[65] LHCb collaboration, Updated measurement of time-dependent CP-violating observables in $B_{s}^{0} \rightarrow J / \psi K^{+} K^{-}$decays, Eur. Phys. J. C 79 (2019) 706 [Erratum ibid. 80 (2020) 601] [arXiv: 1906.08356] [INSPIRE].

[66] ATLAS collaboration, Measurement of the $C P$-violating phase $\phi_{s}$ in $B_{s}^{0} \rightarrow J / \psi \phi$ decays in ATLAS at $13 \mathrm{TeV}$, arXiv:2001.07115 [INSPIRE].

[67] A. Lenz, Theoretical update of B-mixing and lifetimes, in the proceedings of the $47^{\text {th }}$ Rencontres de Moriond on Electroweak Interactions and Unified Theories, March 3-10, La Thuile, Italy (2012), arXiv:1205.1444 [INSPIRE].

[68] A.J. Lenz, A simple relation for $B_{s}$ mixing, Phys. Rev. D 84 (2011) 031501 [arXiv:1106.3200] [INSPIRE].

[69] T. Jubb, M. Kirk, A. Lenz and G. Tetlalmatzi-Xolocotzi, On the ultimate precision of meson mixing observables, Nucl. Phys. B 915 (2017) 431 [arXiv:1603.07770] [INSPIRE].

[70] C. Bobeth and U. Haisch, New Physics in $\Gamma_{12}^{s}:(\bar{s} b)(\bar{\tau} \tau)$ operators, Acta Phys. Polon. B 44 (2013) 127 [arXiv:1109.1826] [INSPIRE].

[71] C. Bobeth, U. Haisch, A. Lenz, B. Pecjak and G. Tetlalmatzi-Xolocotzi, On new physics in $\Delta \Gamma_{d}$, JHEP 06 (2014) 040 [arXiv:1404.2531] [INSPIRE].

[72] C. Cornella, G. Isidori, M. König, S. Liechti, P. Owen and N. Serra, Hunting for $B^{+} \rightarrow K^{+} \tau^{+} \tau^{-}$imprints on the $B^{+} \rightarrow K^{+} \mu^{+} \mu^{-}$dimuon spectrum, Eur. Phys. J. $C \mathbf{8 0}$ (2020) 1095 [arXiv:2001.04470] [INSPIRE].

[73] N. Cabibbo, Unitary symmetry and leptonic decays, Phys. Rev. Lett. 10 (1963) 531 [Erratum ibid. 10 (1963) 648].

[74] M. Kobayashi and T. Maskawa, CP violation in the renormalizable theory of weak interaction, Prog. Theor. Phys. 49 (1973) 652.

[75] R.N. Lee, Presenting LiteRed: a tool for the Loop InTEgrals REDuction, arXiv:1212.2685 [INSPIRE].

[76] R.N. Lee, LiteRed 1.4: a powerful tool for reduction of multiloop integrals, J. Phys. Conf. Ser. 523 (2014) 012059 [arXiv: 1310.1145] [inSPIRE]. 
[77] E. Remiddi and L. Tancredi, Differential equations and dispersion relations for Feynman amplitudes. The two-loop massive sunrise and the kite integral, Nucl. Phys. B 907 (2016) 400 [arXiv: 1602.01481] [INSPIRE].

[78] A.A. Pivovarov, N.N. Tavkhelidze and V.F. Tokarev, The Schwinger model and sum rules, Phys. Lett. B 132 (1983) 402.

[79] S. Groote and A.A. Pivovarov, Threshold expansion of Feynman diagrams within a configuration space technique, Nucl. Phys. B 580 (2000) 459 [hep-ph/0003115] [INSPIRE].

[80] J. Broedel, C. Duhr, F. Dulat and L. Tancredi, Elliptic polylogarithms and iterated integrals on elliptic curves. Part I: general formalism, JHEP 05 (2018) 093 [arXiv: 1712.07089] [INSPIRE].

[81] J. Broedel, C. Duhr, F. Dulat and L. Tancredi, Elliptic polylogarithms and iterated integrals on elliptic curves II: an application to the sunrise integral, Phys. Rev. D 97 (2018) 116009 [arXiv: 1712.07095] [INSPIRE].

[82] M. Ciuchini, E. Franco, V. Lubicz and F. Mescia, Next-to-leading order QCD corrections to spectator effects in lifetimes of beauty hadrons, Nucl. Phys. B 625 (2002) 211 [hep-ph/0110375] [INSPIRE].

[83] P. Gambino, G. Ossola and N. Uraltsev, Hadronic mass and $Q^{2}$ moments of charmless semileptonic B decay distributions, JHEP 09 (2005) 010 [hep-ph/0505091] [INSPIRE].

[84] T. Mannel, D. Moreno and A. Pivovarov, Heavy quark expansion for heavy hadron lifetimes: completing the $1 / m_{b}^{3}$ corrections, JHEP 08 (2020) 089 [arXiv: 2004.09485] [INSPIRE].

[85] P. Gambino, K.J. Healey and S. Turczyk, Taming the higher power corrections in semileptonic B decays, Phys. Lett. B 763 (2016) 60 [arXiv:1606.06174] [INSPIRE].

[86] B. Blok, R. Dikeman and M.A. Shifman, Calculation of $1 / m_{c}^{3}$ terms in the total semileptonic width of D mesons, Phys. Rev. D 51 (1995) 6167 [hep-ph/9410293] [InSPIRE]. 